\title{
COVID-19 mRNA vaccine induced antibody responses and neutralizing antibodies against three SARS-CoV-2 variants
}

\section{Pinja Jalkanen ( $\nabla$ piroja@utu.fi )}

University of Turku

\section{Pekka Kolehmainen}

University of Turku

\section{Hanni Häkkinen}

Helsinki University Hospital and University of Helsinki

\section{Moona Huttunen}

University of Turku https://orcid.org/0000-0002-4745-0477

\section{Paula Tähtinen}

Turku University Hospital and University of Turku

\section{Rickard Lundberg}

University of Turku

\section{Sari Maljanen}

University of Turku

\section{Arttu Reinholm}

University of Turku

\section{Sisko Tauriainen}

University of Turku

\section{Sari Pakkanen}

Helsinki University Hospital and University of Helsinki

\section{Iris Levonen}

Helsinki University Hospital and University of Helsinki

\section{Arttu Nousiainen}

Helsinki University Hospital and University of Helsinki

\section{Taru Miller}

Helsinki University Hospital and University of Helsinki

\section{Hanna Välimaa}

Helsinki University Hospital and University of Helsinki

\section{Lauri Ivaska}

Turku University Hospital and University of Turku https://orcid.org/0000-0001-8935-5032

\section{Arja Pasternack}


University of Helsinki

\section{Rauno Naves}

University of Helsinki

\section{Olli Ritvos}

Haartman Institute, University of Helsinki, and HUSLAB

\section{Pamela Österlund}

Finnish Institute for Health and Welfare https://orcid.org/0000-0002-2229-6661

\section{Suvi Kuivanen}

University of Helsinki

\section{Teemu Smura}

University of Helsinki

Jussi Hepojoki

University of Helsinki https://orcid.org/0000-0001-5699-214X

\section{Olli Vapalahti}

University of Helsinki

\section{Johanna Lempainen}

Turku University Hospital and University of Turku

\section{Laura Kakkola}

University of Turku

\section{Anu Kantele}

Helsinki University Hospital and University of Helsinki

\section{Ilkka Julkunen}

University of Turku and Turku University Hospital

\section{Article}

Keywords: SARS-CoV-2, mRNA vaccine, antibody responses, SARS-CoV-2 variants

Posted Date: March 23rd, 2021

DOI: https://doi.org/10.21203/rs.3.rs-343388/v1

License: (c) (1) This work is licensed under a Creative Commons Attribution 4.0 International License. Read Full License

Version of Record: A version of this preprint was published at Nature Communications on June 28th, 2021. See the published version at https://doi.org/10.1038/s41467-021-24285-4. 


\section{COVID-19 mRNA vaccine induced antibody responses and}

\section{2 neutralizing antibodies against three SARS-CoV-2 variants}

4 Pinja Jalkanen ${ }^{1, *}$, Pekka Kolehmainen ${ }^{1}$, Hanni K Häkkinen ${ }^{2}$, Moona Huttunen ${ }^{1}$, Paula A. Tähtinen ${ }^{3}$,

5 Rickard Lundberg ${ }^{1}$, Sari Maljanen ${ }^{1}$, Arttu Reinholm ${ }^{1}$, Sisko Tauriainen ${ }^{1}$, Sari H Pakkanen ${ }^{2}$, Iris

6 Levonen $^{2}$, Arttu Nousiainen ${ }^{2}$, Taru Miller ${ }^{2}$, Hanna Välimaa ${ }^{2}$, Lauri Ivaska ${ }^{3}$, Arja Pasternack ${ }^{4}$, Rauno

7 Naves $^{4}$, Olli Ritvos ${ }^{4}$, Pamela Österlund ${ }^{5}$, Suvi Kuivanen ${ }^{6}$, Teemu Smura ${ }^{6}$, Jussi Hepojoki ${ }^{6}$, Olli

8 Vapalahti $^{6}$, Johanna Lempainen ${ }^{1,3}$, Laura Kakkola ${ }^{1}$, Anu Kantele ${ }^{2}$, Ilkka Julkunen ${ }^{1,7}$

9 Affiliations:

$10{ }^{1}$ Institute of Biomedicine, University of Turku, Finland

$11{ }^{2}$ Meilahti Vaccination Research Center, MeVac, Department of Infectious Diseases, Helsinki

12 University Hospital and University of Helsinki, Finland

$13{ }^{3}$ Department of Pediatrics and Adolescent Medicine, Turku University Hospital and University of

14 Turku, Turku, Finland

$15{ }^{4}$ Department of Physiology, University of Helsinki, Finland

$16{ }^{5}$ Finnish Institute for Health and Welfare, Helsinki, Finland

$17{ }^{6}$ Department of Virology, University of Helsinki, Finland

$18{ }^{7}$ Clinical Microbiology, Turku University Hospital, Turku, Finland

19 Equal last author contribution L.K., A.K. and I.J. 
20 *Corresponding authors: Pinja Jalkanen (pinja.r.jalkanen@utu.fi) or Ilkka Julkunen

21 (ilkka.julkunen@utu.fi) 


\section{Abstract}

23 As SARS-CoV-2 has been circulating for over a year, dozens of vaccine candidates are under

24 development or in clinical use. The BNT162b2 mRNA COVID-19 vaccine induces spike protein-

25 specific neutralizing antibodies associated with protective immunity. The emergence of the B.1.1.7 and

26 B.1.351 variants has raised concerns of reduced vaccine efficacy and increased re-infection rates. Here

27 we show, that after the second dose, the sera of BNT162b2-vaccinated health care workers $(n=180)$

28 effectively neutralize the SARS-CoV-2 variant with the D614G substitution and the B.1.1.7 variant,

29 whereas the neutralization of the B.1.351 variant is five-fold reduced. Despite the reduction, $92 \%$ of the

30 vaccinees have a neutralization titre of $>20$ for the B.1.351 variant indicating some protection. The

31 vaccinees' neutralization titres exceeded those of recovered non-hospitalized COVID-19 patients. Our

32 work provides strong evidence that the second dose of the BNT162b2 vaccine induces efficient cross-

33 neutralization of SARS-CoV-2 variants currently circulating in the world. 


\section{Introduction}

36 The emergence and spread of the severe acute respiratory syndrome coronavirus 2 (SARS-CoV-2) has

37 caused a pandemic with over 2.7 million deaths ${ }^{1}$ and rapid development of multiple vaccine

38 candidates $^{2}$. SARS-CoV-2 infection elicits antibodies against spike protein $(\mathrm{S})$ and nucleoprotein $(\mathrm{N})^{3-}$

395 , of which, on the basis of virus challenge studies in animals, the spike protein-specific antibodies are

40 neutralizing and associated with protective immunity ${ }^{6,7}$. In addition, recent studies of COVID-19

41 patients and vaccinees indicate that previous infections and vaccinations are related to a decreased rate

42 of SARS-CoV-2 infections ${ }^{8-10}$. Although the persistence of vaccine-induced antibodies is still not

43 known, infection-induced neutralizing antibodies have remained detectable for at least six months after

44 symptom onset ${ }^{11}$.

45 Currently, European Medicines Agency (EMA) has authorized four vaccines to be used in European

46 Union: two mRNA vaccines (BNT162b2 / Comirnaty by Pfizer-BioNTech and mRNA-1273 by

47 Moderna) and two adenoviral vector-based vaccines (ChAdOx1-S by AstraZeneca-Oxford and

48 COVID-19 Vaccine Janssen by Janssen Biologics B.V. and Janssen Pharmaceutica NV) ${ }^{12}$. All four

49 vaccines aim to generate spike protein-specific antibodies and all have been shown to induce anti-S

50 IgG antibodies with neutralizing activity against the first pandemic SARS-CoV-2 Wuhan Hu-1 variant

51 and against the currently circulating D614G variants ${ }^{13-15}$. The recent emergence of SARS-CoV-2

52 variants of concern, such as B.1.1.7 first identified in the United Kingdom ${ }^{16}$ and B.1.351 first identified

53 in South Africa ${ }^{17}$, has raised concerns about increased virus transmissibility and reduced vaccine

54 efficacy. These two variants of concern are defined by eight to ten amino acid changes or deletions in

55 the spike protein to which vaccine-induced antibodies are targeted ${ }^{17-20}$. Both of these variants are now

56 transmitted in several countries (https://cov-lineages.org/global_report.html). Initial studies reported

57 that antibodies produced in response to vaccination and natural infection neutralize the B.1.1.7 
variant $^{19,21}$, whereas neutralization of the B.1.351 is reduced 8 to 13 -fold ${ }^{18,22,23}$. However, it is still unclear whether the B.1.351 variant can escape from humoral and cell-mediated immunity. Here, we characterize the BNT162b2 vaccine-induced antibody responses among a sequential serum sample cohort of 180 Finnish healthcare workers who, belonging to the group targeted first in Finland, received two doses of COVID-19 vaccine with three weeks interval. SARS-CoV-2 S1-specific IgG, IgA, and IgM antibody responses and neutralization titres for three SARS-CoV-2 variants were determined. We show that two-dose immunization yields high levels of anti-S1 IgG antibodies in 100\% of vaccinees. The second vaccine dose induces antibodies for efficient neutralization of D614G and B.1.1.7. variants, whereas the neutralization titres for B.1.351 are lower.

\section{Results}

\section{Study subjects}

69 The vaccinee group comprised 180 volunteers (115 from TYKS and 65 from HUH), aged 20 to 65 70 years (mean 43 and median 41); 149/180 (83\%) were females (age 20-65 years) and 31/180 (17\%)

71 were males (age 22-60 years). The group of recovered COVID-19 patients comprised 50 volunteers 72 (from HUH), aged 19-93 (mean 43 and median 38); 33 were females and 17 males.

\section{Antibody responses against SARS-CoV-2 S1 and N proteins in vaccinees and}

\section{4 convalescent phase patients}

75 In order to monitor the immunological responses of vaccinees, we collected sequential serum samples (0, 3, 6 weeks) from 180 vaccinated health care workers (HCWs). The first vaccinated HCWs received

77 their first vaccination on 28 December 2020, and the last vaccinations for those HCWs in this study were given on 12 February 2021. Serum samples from 50 non-hospitalized, recovered COVID-19 
patients from 2020 were also included in the analysis. The generation of anti-S1 $\operatorname{IgG}$, $\operatorname{IgA}$, $\operatorname{IgM}$, and total Ig antibodies after vaccination was analyzed with enzyme immunoassay (EIA). The original optical density values in the assay were converted to EIA units to minimize inter-assay variation (Fig. 1A). To verify the EIA results and to differentiate those with a previous or concurrent SARS-CoV-2 infection, sera were also analyzed with $\mathrm{N}$ protein-specific EIA (Fig. 1B).

Before vaccination (0 day sampling) 11/180 (6\%) had anti-S1 IgG antibodies (Supplementary Fig. 1A) indicating that these individuals had undergone a previous SARS-CoV-2 infection. Five of these antiS1 positive participants had also anti-N IgG antibodies. Already after the first dose of the vaccine (3 weeks), vaccinees with prior SARS-CoV-2 infection showed clearly increased levels of anti-S1 IgG antibodies (geometric mean 99). After the second dose of the vaccine (6 weeks), all vaccinees with prior SARS-CoV-2 infection had very high levels of anti-S1 IgG antibodies (geometric mean 109)

\section{0 (Supplementary Fig. 1A).}

Three weeks after the first dose of BNT162b2, vaccinees without prior SARS-CoV-2 infection (169/180, 94\%) developed varying levels of anti-S1 IgG antibodies (geometric mean 47), and moderate levels of anti-S1 IgA and IgM antibodies (Fig. 1A, Table 1). Total Ig levels for S1 ranged from 1 to 98 EIA units. The EIA levels for anti-N antibodies among the vaccinees remained the same as before the vaccination (Fig. 1B, Table 1) indicating the absence of SARS-CoV-2 infections in this study group. Anti-N IgG antibody levels were higher in sera of non-hospitalized COVID-19 patients (geometric mean 17) than of vaccinees (geometric mean 2) (Fig. 1B, Table 1). However, already after the first vaccination dose, the geometric mean of anti-S1 IgG and anti-S1 total Ig antibodies of vaccinees exceeded those of convalescent phase COVID-19 patients, 47 and 37 vs. 20 and 23, respectively (Fig. 1A, Table 1). 
101 Six weeks after the first vaccine dose (three weeks after the second vaccine dose) all participants

102 elicited high levels of anti-S1 IgG antibodies together with a modest increase in anti-S1 IgA and IgM

103 antibody levels (Fig. 1A). The vaccinees did not develop anti-N antibodies following vaccination (Fig.

104 1B). Also, based on anti-N antibodies, only one person got infected with SARS-CoV-2 during the 6 weeks: participant was anti-N and anti-S1 IgG negative at 0 day sampling, and anti-N and anti-S1 IgG

106 positive at 3 week sampling. The negative control antigen signals were close to the background values

107 (Supplementary Fig. 2). Anti-S1 IgG antibody and total anti-S1 Ig levels induced by two doses of

108 BNT162b2 vaccine were clearly higher than the anti-S1 IgG levels measured from the convalescent

109 phase patient sera, geometric means being 107 and 86 vs. 20 and 23, respectively.

\section{Characterization of SARS-CoV-2 isolates}

111 To analyze the neutralization capacity of the vaccinees' sera, we isolated for microneutralization tests

112 four virus variants circulating in Finland: D614G variants FIN-25 (spring 2020) representing B.1

113 lineage and SR121 (autumn 2020) representing B.1.463 lineage, a variant of concern 85HEL

114 representing B.1.1.7 lineage and a variant of concern HEL12-102 representing B.1.351 lineage. FIN-25

115 isolate was passaged first in VeroE6 cells followed by passaging in VeroE6 cells expressing

116 transmembrane protease serine 2 (VeroE6-TMPRSS2-H10). Other three isolates were passaged in

117 VeroE6-TMPRSS2-H10 cells to avoid the generation of mutations in the vicinity of the furin cleavage

118 site (Fig. 2A). The isolates were sequenced to compare the mutations in SR121, 85HEL (B.1.1.7) and

119 HEL12-102 (B.1.351) variants to FIN-25 that represented the circulating strains in Finland until the 120 emergence of variants of concern. Sequence analysis of SARS-CoV-2 isolates revealed 3 amino acid

121 changes in the spike protein of FIN-25, 4 in SR121, 10 in 85HEL (B.1.1.7) and 9 in HEL12-102

122 (B.1.351) variants compared to original Wuhan Hu1 strain (Fig. 2A). The sequence of FIN-25 that was

123 passaged initially in VeroE6 cells had close to the furin cleavage site a deletion of amino acids 674-678 
124 in $45 \%$ and $\mathrm{R} 682 \mathrm{~W}$ mutation at the furin cleavage site in $41 \%$ of the virus population, indicating some

125 heterogeneity of the FIN-25 virus stock, which did, however, not affect the growth properties of the

126 virus (data not shown). Sequences of the three other isolates passaged in VeroE6-TMPRSS2-H10 cells

127 only had either aforementioned deletion in the minority of the virus population (8\% of SR121) or a

128 completely intact furin cleavage site. Otherwise, all spike protein sequences obtained from the virus

129 propagations were identical to the sequences obtained from the respective original patient sample, all

130 also containing the D614G substitution linked to increased fitness and transmissibility ${ }^{24,25}$.

131 The availability of the 3-dimensional structure of SARS-CoV-2 spike protein enabled the positioning

132 of the amino acid changes into the structure of the trimeric spike protein (Fig. 2B). Substitutions found

133 in FIN-25 and SR121 spike proteins localize in the stem regions of the trimeric spike protein. The

134 substitutions found in the spike proteins of B.1.1.7 and B.1.351 variants located both to the stem region

135 and on the surface of the trimeric spike protein close to the receptor binding domain (RBD). The three

136 B.1.351 variant substitutions E484K, K417N, and N501Y are in the groove of RBD - ACE2

137 interaction domain. In addition, both the B.1.1.7 and B.1.351 variants had 3 amino acid deletions in the

138 far edges of the 3-dimensional structure (Fig. 2B). Figure 2C shows combined amino acid changes

139 found in the isolates used in this study indicating the accumulation of substitutions on multiple

140 localizations on the trimeric structure of the spike protein. The amino acid changes in the spike protein,

141 especially aforementioned E484K, K417N, and N501Y have recently been reported to affect the

142 neutralizing efficacy of the antibodies ${ }^{26}$.

\section{Neutralizing antibody titres against SARS-CoV-2 variants}

144 To measure the neutralizing potential of the vaccinees' sera against all four SARS-CoV-2 isolates,

145 neutralizing antibody titres elicited by the BNT162b2 vaccine were analyzed with microneutralization

146 test (MNT). The neutralizing titres with two D614G isolates FIN-25 and SR121 were almost identical 
147 both three weeks $(\mathrm{p}=0.02)$ and six weeks after the first dose $(\mathrm{p}=0.11)($ Fig. 3A), indicating that the

148 mutations in FIN-25 spike protein due to initial propagation in VeroE6 cells did not affect the

149 neutralizing titres.

150 Before vaccination (0 day sampling) those 11/180 with a likely SARS-CoV-2 infection based on EIA 151 results, showed increased geometric mean titres (GMT) of 35, 31 and 16 against FIN-25, 85HEL

152 (B.1.1.7) and HEL12-102 (B.1.351) virus isolates, respectively. Following the first and the second 153 vaccination, the GMTs further increased to 435 and 682, to 320 and 640, and to 101 and 132,

154 respectively (Supplementary Fig. 1B). These results with this small group indicate that even one dose

155 of BNT162b2 vaccine induces high MNT titres in those individuals who had suffered a previous

156 COVID-19 infection.

157 None of the vaccinees without a prior SARS-CoV-2 infection (169/180) had neutralizing antibodies

158 before the vaccination (Fig. 3A). Three weeks after the first vaccine dose, neutralizing titres against all

159 four isolates were slightly increased (GMT of 24 for FIN-25, 32 for SR121, 24 for 85HEL (B.1.1.7)

160 and 12 for HEL12-102 (B.1.351)). Six weeks after the first dose of the vaccine (three weeks after the

161 second dose), neutralizing titres were increased to a GMT of 234 against FIN-25, 275 against SR121,

162240 against 85HEL (B.1.1.7) and 48 against HEL12-102 (B.1.351) (Fig. 3A, Table 1). Three weeks

163 after the first dose $37 \%, 17 \%, 37 \%$ and $85 \%$ of vaccinees had a neutralization titre <20 against FIN-25,

164 SR121, 85HEL (B.1.1.7) and HEL-12-102 (B.1.351) isolates, respectively. After the second vaccine

165 dose, $100 \%$ of vaccinees had neutralizing antibodies against FIN-25, SR121 and 85HEL (B.1.1.7),

166 whereas $92 \%$ of vaccinees had neutralizing antibodies against the HEL-12-102 (B.1.351) variant.

167 GMTs against all four isolates in vaccinees exceeded the GMTs seen in convalescent phase patient sera 168 (Fig. 3A, Table 1). 
170 ( $\mathrm{p}<0.0001)$ compared to FIN-25 and 85HEL (B.1.1.7). After the second immunization, the GMT for

171 HEL-12-102 (B.1.351) was 5-fold lower compared to FIN-25 and 85HEL (B.1.1.7) (Fig. 3B).

172 The MNT titres for two D614G-containing isolates, FIN-25 and SR121, correlated very well, as also 173 did FIN-25 and 85HEL (B.1.1.7) (r>0.8, p<0.0001) (Fig. 4). MNT titres for FIN-25 and HEL-12-102

174 (B.1.351) correlated relatively well and highly significantly $(\mathrm{r}=0.74, \mathrm{p}<0.0001)$, as did the two variants

175 of concern, 85HEL (B.1.1.7) and HEL-12-102 (B.1.351) $(\mathrm{r}=0.75, \mathrm{p}<0.0001)$.

176 To analyze the effect of age and gender to the antibody responses, the vaccinees were divided into age

177 and gender groups and the S1 IgG EIA and MNT results were compared between the groups (Fig. 5A and B). After the first vaccine dose, anti-S1 IgG antibody levels and neutralization titres decreased

179 significantly in the older age group (55-65 years) compared to younger age groups (20-34 and 35-44

180 years) (Fig. 5A). However after the second vaccine dose, the neutralization titres were similar between

181 the age groups (GMT 257, 268, 200 and 206 in age groups of 20-34, 35-44, 45-54 and 55-65 years,

182 respectively) (Fig. 5A). We also compared gender-related antibody responses even though male

183 vaccinees were underrepresented, comprising only 17\% (29/169) of the vaccinees. After the second

184 dose, female vaccinees had slightly higher neutralization titres than male $(\mathrm{p}=0.0412)$, although the anti-

185 S1 IgG antibody levels remained at the same level (Fig. 5B).

\section{EIA values correlate with MNT titres}

187 Neutralization tests with live SARS-CoV-2 viruses are very time-consuming, and at the moment the 188 assay requires BSL-3 laboratory conditions, whereas EIA and other similar colorimetric/fluorometric 189 antibody assays are faster and user friendlier. To assess whether EIA values correlate with MNT titres, 190 anti-S1 IgG and total anti-S1 Ig were compared to neutralization titres against FIN-25 (Fig. 6,

191 Supplementary Fig. 3). Both anti-S1 IgG and total anti-S1 Ig EIA measurements correlated very well 
192 with MNT titres $(r>0.9, p<0.0001)$ suggesting that EIA, especially IgG EIA, using spike protein as an

193 antigen could be a useful method to determine COVID-19 immunity.

\section{Discussion}

195 The emergence of the COVID-19 pandemic in early 2020 prompted a rapid development of various

196 types of vaccines such as mRNA encoding SARS-CoV-2 spike protein, viral vector based (e.g.

197 adenovirus), inactivated virus, virus-like particle, and recombinant protein vaccines. Once the

198 European Union had made agreements with a number of vaccine producers, mass immunization was

199 started in Finland at the end of December 2020, first with the mRNA based Pfizer-BioNTech vaccine

200 and somewhat later the Moderna mRNA and AstraZeneca adenovirus-based vaccines ${ }^{12}$. Vaccination of

201 health care professionals within a national vaccination programme in Finland enabled us to start

202 independent of pharmaceutical companies a follow-up study of vaccine-induced immunity. In the

203 present report, we show that two-dose vaccination with the BNT162b2 mRNA COVID-19 vaccine

204 induces very high antibody levels against viral spike protein and high titres of neutralizing antibodies.

205 The vaccine induced good cross-reactivity to D614G and B.1.1.7 variants and, albeit reduced levels,

206 detectable neutralizing antibodies to B.1.351 variant.

207 EIA is a rapid and sensitive method to analyze immune responses against vaccine antigens or different

208 viral proteins in response to infection. The method is easily quantitative and suitable for analyzing

209 different immunoglobulin classes. In this study, we observed that practically all seronegative health

210 care workers (20-65 years of age) responded to the first BNT162b2 vaccine dose and an increase in

211 spike protein-specific antibody responses in $\operatorname{IgG}$ antibody class was detectable. The antibody levels

212 varied considerably, however, and relatively few individuals showed increased antibody levels in the

213 IgA and IgM antibody classes. The second vaccine dose, which was given according to the original 
214 vaccination protocol three weeks after the first vaccine dose, induced very high levels of spike protein215 specific $\operatorname{IgG}$ antibodies, while $\operatorname{Ig} \mathrm{A}$ and $\operatorname{IgM}$ responses remained low. The vaccinees' IgG antibody 216 levels were on average higher than measured in convalescent phase sera from home-treated patients. 217 Antibody responses have been found generally higher for COVID-19 patients with a more severe 218 disease ${ }^{23,27,28}$, however, as shown by this study also, BNT162b2 vaccine appears to induce higher 219 antibody levels than those measured in patients ${ }^{29,30}$. Remarkably, administration of two doses of the 220 mRNA vaccine induced very high antibody responses in $100 \%$ of the vaccinees.

221 The global circulation of SARS-CoV-2 and a huge number of infections worldwide have led to the 222 emergence of hundreds of evolutionary lineages and variants of the virus (https://cov-

223 lineages.org/global_report.html). The evolutionary speed of SARS-CoV-2 has been relatively slow, at 224 least compared to influenza A viruses, presumably due to a virus-encoded enzyme with proof-reading 225 capability. Within the first 15 months of circulation, up to 30-35 mutations have been identified 226 accumulating into the viral genome. Many of these mutations are silent or appear in places of the 227 genome that are not critical for avoiding immunity induced by vaccination or natural infection. 228 However, a number of variants have raised concern due to mutations accumulating particularly in the 229 S-gene and causing changes in the immunodominant epitopes of the trimeric spike protein. Mapping 230 the spike protein mutations on variants sequenced and used in this study revealed that they occur 231 outside the globular head of the trimeric spike protein. The D614G and B.1.1.7 variant viruses were 232 readily neutralized by the vaccinees' sera, indicating that these mutations are unlikely to impair the 233 neutralizing antibody capacity induced by vaccination or natural infection. However, it should be noted 234 that the neutralizing titre of these sera decreased five-fold against the B.1.351variant, which denotes 235 that the amino acid changes accumulating in this variant are potentiating the escape of the virus from 236 the humoral immune responses. Despite this, more than $92 \%$ of the vaccinees showed measurable 
237 neutralizing antibody titres against the B.1.351 variant, suggesting that the spike protein encoded by 238 Pfizer-BioNTech's mRNA vaccine is similar enough to also mount an immune response against the 239 B.1.351 variant.

240 The critical amino acid changes linked to escape from humoral immunity in the B.1.351 variant appear 241 to be K417N, E484K, and N501Y $\mathrm{Y}^{30-32}$. These amino acids are situated in the grooves within the 242 receptor binding site of the trimeric S protein complex. There is no three-dimensional structure 243 presently available for the B.1.351variant spike protein trimer, but because of its relatively radical 244 amino acid substitutions, conformational changes in the spike structure may prove substantial. 245 Interestingly, the B.1.351 and B.1.1.7 variants have deletions in the tips of the globular S1 domain 246 (amino acids 243-245 and amino acids 69-70 and 244, respectively) which could contribute to the 247 impaired recognition by neutralizing antibodies.

248 It is currently not known how high neutralizing antibody titres against a given virus variant are required 249 for antibody-mediated protection against the COVID-19. However, the clinical efficacy data 250 accumulating from COVID-19 vaccine studies strongly suggest that already one dose of the vaccine 251 provides protection against severe COVID-19, even when neutralizing antibody levels cannot be 252 detected in all vaccinees ${ }^{33,34}$. This suggests that the first vaccine dose may prime the individual for a 253 rapid induction of protective immunity when contracting the virus in nature and avoiding severe 254 COVID-19. According with previous data ${ }^{29,35-38}$, we found that individuals with prior SARS-CoV-2 255 infection readily responded to the first vaccine dose with high antibody levels and neutralization titres.

256 Humoural immune response to vaccinations has been shown to decline with age ${ }^{39,40}$. Consistently, we 257 observed a trend of declining immune response to the COVID-19 mRNA vaccine by age. This trend 258 was not very strong, presumably because the ages of our vaccinees ranged from 20 to 65 years, while 259 age-dependent immunosenescence should be more pronounced in the age group $>65$ years ${ }^{39}$. Another 
explanation might be that the BNT162b2 mRNA vaccine is exceptionally immunogenic and therefore, 261 especially when given two doses, it enables practically all individuals regardless of gender and age, to 262 develop high antibody levels and neutralization titres.

263 In summary, in the present study we show that the Pfizer-BioNTech BNT162b2 COVID-19 mRNA 264 vaccine is highly immunogenic, and particularly after two vaccine doses all vaccinees showed very 265 high humoral immune response to D614G variant viruses. Immunity to a recent B.1.1.7 variant was 266 equally good as compared to the D614G variant, whereas vaccine- and SARS-CoV-2- infection 267 induced immunity against B.1.351 variant was reduced. Despite this, almost all vaccinees showed 268 neutralizing antibodies against the B.1.351 variant, suggesting to provide at least some degree of 269 protection against these variant viruses. In the future, it will be intriguing to study the development and 270 persistence of cell-mediated immunity induced by COVID-19 vaccines. Promising data has been 271 reported at least for the BNT162b2 vaccine which in preliminary studies has induced good cell272 mediated immunity ${ }^{41,42}$. As the use of other types of SARS-CoV-2 vaccines will be increased, it is the 273 responsibility of the scientific community and public health professionals to systematically collect 274 serum and cellular samples for comparative analyses of vaccine-induced immunity, cross-protection 275 and longevity of vaccine-and natural infection-induced immunity.

276 As a whole, all vaccines that have currently obtained market authorization in EU show excellent 277 protective efficacy against severe COVID-19. Thus, it is very likely that immunogenicity results 278 similar to those presented here will be applicable to them as well. The future of SARS-CoV-2 vaccines 279 therefore seems to look bright. 
280 Methods

\section{Study participants}

282 SARS-CoV-2 vaccinations started in Finland at the end of December 2020 with Pfizer-BioNTech 283 BNT162b2 mRNA (Comirnaty) vaccine. Study participants $(\mathrm{n}=180)$ were recruited among healthcare 284 personnel of Turku University Hospital (TYKS, Turku, Finland) (Southwest Finland health district 285 ethical permission ETMK 19/1801/2020) and Helsinki University Hospital (HUH, Helsinki, Finland) 286 (Helsinki-Uusimaa health district ethical permission HUS/1238/2020) prior to receiving an optimal 287 regimen of two doses of BNT162b2 mRNA vaccine at a three-week dosing interval as part of hospital 288 occupational health care. Serum samples were collected before or on the day of the first vaccine dose (0 289 day sample, $n=180$ ), 16 to 28 days (mean 20) after the first vaccine dose ( 3 week sample, $n=176$ ), and 29013 to 33 (mean 23) days after the second vaccine dose (34 to 54 days after the first vaccine dose) (6 291 week sample, $\mathrm{n}=180$ ).

292 Convalescent phase serum samples $(\mathrm{n}=50)$ were collected at HUH from patients with initial RT-qPCR 293 confirmed home-treated COVID-19 infection (Helsinki-Uusimaa health district ethical permission 294 HUS/1238/2020). The patients provided written informed consent and were sampled 14 days -6 295 weeks after the positive PCR test result. As negative control serum samples ( $\mathrm{n}=40)$ we used randomly 296 selected diagnostic serum samples collected at TYKS prior to COVID-19 pandemic (Jalkanen et al. 297 2020, submitted).

\section{Expression and purification of SARS-CoV-2 nucleoprotein and S1 antigens}

SARS-CoV-2 protein expression was done as described previously (Jalkanen et al. 2020, submitted). 
302 (N-GST) in Spodoptera frugiperda (Sf-9) cells. GST alone was produced to be used as a control

303 protein. S1 domain of the spike protein (amino acid residues 16-541) was expressed as a fusion protein

304 with mouse IgG2a Fc and 8xhistidine tag (S1-mFc-8xhis) in human embryonic kidney (HEK293F)

305 cells. Mouse promyostatin (ProMstn)-mFc(IgG2a)-6xhis (later referred as Mstn-mFc) was produced to

306 be used as a control protein. Proteins were purified and buffer was exchanged to PBS. Concentrations

307 of produced proteins were measured with BCA protein assay kit (Thermo Fisher Scientific).

308 IgG, IgA, and IgM EIA and total Ig EIA for SARS-CoV-2 S1 and N protein

309 antibodies

310 Enzyme immunoassay (EIA) was performed by coating 96-well microtitre plates (Nunc Maxisorp,

311 Thermo Fisher Scientific) for $16 \mathrm{~h}$ at $+4^{\circ} \mathrm{C}$ with GST-N $(2.0 \mu \mathrm{g} / \mathrm{ml}), \mathrm{S} 1-\mathrm{mFc}(3.5 \mu \mathrm{g} / \mathrm{ml})$, and

312 corresponding molar amounts of GST $(0.7 \mu \mathrm{g} / \mathrm{ml})$ and Mstn-mFc $(2.4 \mu \mathrm{g} / \mathrm{ml})$ antigens in PBS. The

313 plates were washed with $0.1 \%$ Tween-20 in PBS and blocked for 30 min with assay buffer (5\% swine

314 serum (BioInd), $0.1 \%$ Tween-20 in PBS) before the addition of $50 \mu 1$ serum dilutions (final dilution

$3151: 300$ or $1: 1000$ for some analyses in assay buffer). After $2 \mathrm{~h}$ incubation at $+37^{\circ} \mathrm{C}$, the plates were

316 washed three times followed by addition of $100 \mu \mathrm{l}$ horseradish peroxidase (HRP) conjugated anti-

317 human antibodies (1:8000 dilution of anti-hIgG HRP (Dako A/S), 1:8000 dilution of anti-hIgA HRP

318 (Invitrogen), 1:4000 dilution of anti-hIgM HRP (Dako A/S), and 1:20 000 dilution of anti-hIg (IgG,

$319 \operatorname{IgA}, \operatorname{IgM}) \mathrm{HRP}(\mathrm{Abcam}))$ for $1 \mathrm{~h}$ at $+37^{\circ} \mathrm{C}$. The plates were washed three times and $100 \mu \mathrm{lMB}$ One

320 substrate (Kementec Solutions A/S) was added. The plates were incubated for $20 \mathrm{~min}$ at room

321 temperature, $100 \mu \mathrm{l}$ of $0.2 \mathrm{~N}$ sulphuric acid was added to stop the reaction and the levels of $\operatorname{IgG}, \operatorname{IgA}$,

$322 \mathrm{IgM}$, and total Ig antibodies were measured at $450 \mathrm{~nm}$ with Victor Nivo plate reader (Perkin Elmer).

323 Optical density (OD) values were converted into EIA units by comparing the sample OD values to the 
324 OD values of positive (marked as 100) and negative control samples (marked as 0). EIA units <1 were

325 marked as 1. Cut-off units for S1-based EIA were calculated as the average of 20 negative samples plus 326 three standard deviations (SDs) and for N-based EIA as he average of 20 negative samples plus six 327 SDs.

\section{Propagation of SARS-CoV-2 isolates}

SARS-CoV-2 isolate Fin/25/20 (Gisaid: EPI_ISL_412971) from lineage B.1 was isolated from the nasopharyngeal sample of COVID-19 patient in Finland in February 2020. Swab sample in transport medium was inoculated onto African green monkey kidney epithelial VeroE6 cells at $+37^{\circ} \mathrm{C}$ and $5 \%$ $\mathrm{CO}_{2}$ in culture medium (Eagle's minimum essential medium (EMEM) supplemented with $2 \%$ fetal bovine serum (FBS), $0.6 \mu \mathrm{g} / \mathrm{mL}$ penicillin, $60 \mu \mathrm{g} / \mathrm{mL}$ streptomycin, $2 \mathrm{mM} \mathrm{L}$-glutamine, $20 \mathrm{mM}$ HEPES). Virus was propagated in VeroE6 cells for a total of three times. Subsequently, a VeroE6 clone expressing TMPRSS2, a serine protease essential for SARS-CoV-2 spike protein integrity, was generated (VeroE6-TMPRSS2-H10) ${ }^{43}$. Fin/25/20 was further propagated twice in VeroE6-TMPRSS2H10 cell line (virus isolate named FIN-25). Another 2020 isolate, SR121 from lineage B.1.463, isolated from a patient in Finland in September 2020, was isolated and propagated only in VeroE6-TMPRSS2H10 cells ${ }^{43}$. Variants 85HEL of B.1.1.7 lineage and HEL12-102 of B.1.351 lineage were isolated from patients in Finland as described ${ }^{23}$ and further propagated only in VeroE6-TMPRSS2-H10 cells.

VeroE6-TMPRSS2-H10 cells were maintained in D-MEM (Lonza) supplemented with 10\% FBS, 2mM L-glutamine (Gibco) and penicillin/streptomycin. For virus propagation in VeroE6-TMPRSS2-H10 cells, D-MEM supplemented with 2\% FBS, 2 mM L-glutamine and penicillin/streptomycin was used.

344 Supernatants containing viruses were harvested, cell debris removed with centrifugation at $500 \mathrm{~g}$ for 5 $345 \mathrm{~min}$, and aliquots stored at $-80^{\circ} \mathrm{C}$. 
346 Fifty-percent tissue culture infective dose $\left(\mathrm{TCID}_{50}\right)$ of virus stocks was determined with endpoint 347 dilution assay in VeroE6-TMPRSS2-H10 cells. Briefly, 50000 cells per well were plated on 96-well 348 tissue culture plates (Sarstedt), and the next day media was changed to infection media (2\% FBS). Ten349 fold virus dilutions in infection media were applied onto cells, and the plates were incubated for 3 days 350 at $+37^{\circ} \mathrm{C}$ and $5 \% \mathrm{CO}_{2}$. Cells were fixed with $4 \%$ formaldehyde and stained with crystal violet. Virus 351 dilution resulting in 50\% cell death was determined to represent $\mathrm{TCID}_{50}$ value of the stock virus. Virus 352 propagations and end point dilution assays were done in BSL-3 laboratory conditions.

\section{Sequencing of SARS-CoV-2 isolates}

354 For sequencing of virus stocks, the viral RNA was extracted from supernatants using the RNeasy Mini 355 kit (Qiagen) and reverse-transcribed to cDNA with LunaScript RT SuperMix kit (New England 356 Biolabs). Primer pools targeting SARS-CoV-2 were designed using PrimalScheme tool ${ }^{44}$ and PCR was 357 done with PhusionFlash PCR master mix (Thermo Scientific). Sequencing libraries were prepared with 358 NEBNext ultra II FS DNA library kit (New England Biolabs) according to the manufacturer's 359 instructions and sequenced using Illumina Miseq with v3 sequencing kit. Raw sequence reads were 360 trimmed, and low quality (quality score $<30$ ) and short ( $<25 \mathrm{nt})$ sequences were removed using

361 Trimmomatic ${ }^{45}$. The trimmed sequence reads were assembled to the reference sequence 362 (NC_045512.2) using BWA-MEM ${ }^{46}$ algorithm implemented in SAMTools version $1.8^{47}$. Sequences of 363 four SARS-CoV-2 isolates used in this study were deposited in GenBank: FIN-25 (GenBank 364 MW717675), SR121 (GenBank MW717676), 85HEL (GenBank MW717677) and HEL-12-102 365 (GenBank MW717678). 


\section{Illustration of amino acid changes in SARS-CoV-2 spike protein}

367 SARS-CoV-2 spike structure in closed conformation obtained through cryo-electron microscopy, pdb 368 accession $6 \mathrm{VXX}^{48}$, was used for illustration of the residue differences between the employed SARS-

369 CoV-2 variants in YASARA (available at http://www.yasara.org/).

\section{Microneutralization test}

371 Neutralizing antibodies were measured using a microneutralization test (MNT). Serum samples were 372 serially diluted two-fold, starting at 1:20 dilution, in 2\% FBS in DMEM and incubated with 100

373 TCID 50 of SARS-CoV-2 isolate in $96-$ well tissue culture plates (Sarstedt) for $1 \mathrm{~h}$ at $+37^{\circ} \mathrm{C}$. VeroE6-

374 TMPRSS2-H10 cells were added (40 000-50 000 cells per well) and the plates were incubated at $375+37^{\circ} \mathrm{C}, 5 \% \mathrm{CO}_{2}$ for 3 days. Cells were fixed with $4 \%$ formaldehyde and stained with crystal violet. 376 MNT titres were calculated as the reciprocal dilution resulting in 50\% inhibition of cell death. MNT 377 assays were done at the BSL-3 laboratory conditions.

\section{Statistical analysis}

379 Geometric means with geometric standard deviations (SD) were calculated with GraphPad Prism 8 380 software. Statistical significance of differences between variants were analyzed with Wilcoxon 381 matched pairs signed-rank test, and two-tailed p-values $<0.05$ were considered significant. Differences 382 between age and gender groups were tested with two-tailed Mann-Whitney U test.

\section{References}

384 1. Dong, E., Du, H. \& Gardner, L. An interactive web-based dashboard to track COVID-19 in real 385 time. Lancet Infect. Dis. 20, 533-534 (2020).

386 2. Dong, Y. et al. A systematic review of SARS-CoV-2 vaccine candidates. Signal Transduct. 387 Target. Ther. 5, 237 (2020). 
388

389

390

391

392

393

394

395

396

397

398

399

400

401

402

403

404

405

406

407

408

409

410

411

412

413

414

415

416

417

418

419

420

421

422

423

424

425

426

3. Okba, N. M. A. et al. Severe Acute Respiratory Syndrome Coronavirus 2 - Specific Antibody Responses in Coronavirus Disease Patients. Emerg infec dis 26, 1478-1488 (2020).

4. Amanat, F. et al. A serological assay to detect SARS-CoV-2 seroconversion in humans. Nat. Med. 26, 1033-1036 (2020).

5. Wajnberg, A. et al. Robust neutralizing antibodies to SARS-CoV-2 infection persist for months. Science (80-. ). 370, 1227-1230 (2020).

6. Deng, W. et al. Primary exposure to SARS-CoV-2 protects against reinfection in rhesus macaques. Science (80-. ). 369, 818-823 (2020).

7. Alsoussi, W. B. et al. A Potently Neutralizing Antibody Protects Mice against SARS-CoV-2 Infection. J. Immunol. 205, 915-922 (2020).

8. Hanrath, A. T., Payne, B. A. I. \& Duncan, C. J. A. Prior SARS-CoV-2 infection is associated with protection against symptomatic reinfection. J. Infect. 2020-2021 (2020).

9. V, H. et al. Do antibody positive healthcare workers have lower SARS-CoV-2 infection rates than antibody negative healthcare workers? Large multi-centre prospective cohort study (the SIREN study), England: June to November 2020. medRxiv (2021). doi:10.2139/ssrn.3768524

10. Chodick, G. et al. The effectiveness of the first dose of BNT162b2 vaccine in reducing SARSCoV-2 infection 13-24 days after immunization: real-world evidence. medRxiv (2021).

11. Pradenas, E. et al. Stable neutralizing antibody levels 6 months after mild and severe COVID-19 episodes. Med 2, 313-320 (2021).

12. EMA. COVID-19 vaccines authorised European Medicines Agency. https://www.ema.europa.eu/en/human-regulatory/overview/public-health-threats/coronavirusdisease-covid-19/covid-19-latest-updates

13. Folegatti, P. M. et al. Safety and immunogenicity of the ChAdOx1 nCoV-19 vaccine against SARS-CoV-2: a preliminary report of a phase $1 / 2$, single-blind, randomised controlled trial. Lancet 396, 467-478 (2020).

14. Anderson, E. J. et al. Safety and Immunogenicity of SARS-CoV-2 mRNA-1273 Vaccine in Older Adults. N. Engl. J. Med. 383, 2427-2438 (2020).

15. Walsh, E. E. et al. Safety and Immunogenicity of Two RNA-Based Covid-19 Vaccine Candidates. N. Engl. J. Med. 383, 2439-2450 (2020).

16. Investigation of SARS-CoV-2 variants of concern in England. (2021). https://www.gov.uk/government/publications/investigation-of-novel-sars-cov-2-variant-variantof-concern-20201201

17. Tegally, H. et al. Emergence and rapid spread of a new severe acute respiratory syndrome-related coronavirus 2 (SARS-CoV-2) lineage with multiple spike mutations in South Africa. medRxiv (2020).

18. Zhou, D. et al. Evidence of escape of SARS-CoV-2 variant B.1.351 from natural and vaccine induced sera. Cell (2021).

19. Xiaoying Shen et al. SARS-CoV-2 variant B.1.1.7 is susceptible to neutralizing antibodies elicited by ancestral Spike vaccines. Cell Host Microbe (2021). 
20. Rambaut, A. et al. Preliminary genomic characterisation of an emergent SARS-CoV-2 lineage in the UK defined by a novel set of spike mutations. Virological.org (2020).

21. Muik, A. Neutralization of SARS-CoV-2 lineage B.1.1.7 pseudovirus by BNT162b2 vaccineelicited human sera. Science (80-. ). 6105, 1-5 (2021).

22. Cele, S. et al. Escape of SARS-CoV-2 501Y.V2 variants from neutralization by convalescent plasma. medRxiv (2021).

23. Virtanen, J. et al. Reduced neutralization of B . 1 . 351 variant SARS-CoV-2 by convalescent sera of COVID-19 patients. Res. Sq. (2021).

24. Plante, J. A. et al. Spike mutation D614G alters SARS-CoV-2 fitness. Nature (2020).

25. Hou, Y. J. et al. SARS-CoV-2 D614G variant exhibits enhanced replication ex vivo and earlier transmission in vivo. Science (80-. ). 370, 1464-1468 (2021).

26. Wibmer, C. K. et al. SARS-CoV-2 501Y . V2 escapes neutralization by South African COVID19 donor plasma. Nat. Med. (2021).

27. Lynch, K. L. et al. Magnitude and Kinetics of Anti-Severe Acute Respiratory Syndrome Coronavirus 2 Antibody Responses and Their Relationship to Disease Severity. Clin. Infect. Dis. 72, 301-308 (2021).

28. Grossberg, A. N. et al. A multiplex chemiluminescent immunoassay for serological profiling of COVID-19-positive symptomatic and asymptomatic patients. Nat. Commun. 12, (2021).

29. Manisty, C. et al. Correspondence Antibody response to first BNT162b2 dose in previously SARS-CoV-2-infected individuals. Lancet 6736, 2-3 (2021).

30. Wang, Z. et al. mRNA vaccine-elicited antibodies to SARS-CoV-2 and circulating variants. Nature 1-51 (2021).

31. Collier, D. A. et al. Sensitivity of SARS-CoV-2 B.1.1.7 to mRNA vaccine-elicited antibodies. Nature (2021).

32. Liu, Y. et al. Neutralizing Activity of BNT162b2-Elicited Serum. N. Engl. J. Med. (2021).

33. Dagan, N. et al. BNT162b2 mRNA Covid-19 Vaccine in a Nationwide Mass Vaccination Setting. N. Engl. J. Med. 1-12 (2021).

34. Amit, S., Regev-Yochay, G., Afek, A., Kreiss, Y. \& Leshem, E. Early rate reductions of SARSCoV-2 infection and COVID-19 in BNT162b2 vaccine recipients. Lancet (2021).

35. Krammer, F. et al. Antibody Responses in Seropositive Persons after a Single Dose of SARSCoV-2 mRNA Vaccine. N. Engl. J. Med. (2021).

36. Saadat, S. et al. Binding and Neutralization Antibody Titers After a Single Vaccine Dose in Health Care Workers Previously Infected With SARS-CoV-2. JAMA (2021).

37. Polack, F. P. et al. Safety and Efficacy of the BNT162b2 mRNA Covid-19 Vaccine. N. Engl. J. Med. 383, 2603-2615 (2020).

38. Capetti, A. F. et al. Impressive boosting of anti-S1/S2 IgG production in COVID-19-experienced patients after the first shot of the BNT162b2 mRNA COVID-19 Vaccine. Clin. Infect. Dis. (2021).

39. Chen, W. H. et al. Vaccination in the elderly: an immunological perspective. Trends Immunol. 30, 
40. Prendecki, M. et al. Effect of previous SARS-CoV-2 infection on humoral and T-cell responses to single-dose BNT162b2 vaccine. Lancet 6736, 10-12 (2021).

468

469

470

471

472

473

474

475

476

477

478

479

480

481

482

483

484

485

41. Sahin, U. et al. COVID-19 vaccine BNT162b1 elicits human antibody and TH1 T cell responses. Nature 586, 594-599 (2020).

42. Goel, R. R., Apostolidis, S. A., Painter, M. M. \& Mathew, D. Longitudinal Analysis Reveals Distinct Antibody and Memory B Cell Responses in SARS-CoV2 Naïve and Recovered Individuals Following mRNA Vaccination. medRxiv (2021).

43. Rusanen, J. et al. A generic, scalable, and rapid TR-FRET -based assay for SARS-CoV-2 antigen detection. medRxiv 1-35 (2020).

44. Quick, J. et al. Multiplex PCR method for MinION and Illumina sequencing of Zika and other virus genomes directly from clinical samples. Nat. Protoc. 12, 1261-1266 (2017).

45. Bolger, A. M., Lohse, M. \& Usadel, B. Trimmomatic: a flexible trimmer for Illumina sequence data. Bioinformatics 30, 2114-2120 (2014).

46. Li, H. Aligning sequence reads, clone sequences and assembly contigs with BWA-MEM. (2013).

47. Li, H. et al. The Sequence Alignment/Map format and SAMtools. Bioinformatics 25, 2078-2079 (2009).

48. Walls, A. C. et al. Structure, Function, and Antigenicity of the SARS-CoV-2 Spike Glycoprotein. Cell 181, 281-292.e6 (2020). 
487 We thank Soili Jussila for cell maintenance, Mikael Ritvos for assistance in protein production, and 488 Anne Suominen and Anne-Mari Pieniniemi for technical assistance. I.J. was funded by Jane and Aatos 489 Erkko Foundation (grant numbers 3067-84b53 and 5360-cc2fc), the Academy of Finland (AoF; grant 490 number 336410, 337530) and Sigrid Jusélius Foundation. JH was funded by AoF (grant numbers 491 308613) A.K. was funded by AoF (grant numbers 336439 and 335527), the Finnish Medical 492 Foundation, and private donors through UH. O.V, T.S and S.K were funded by Jane and Aatos Erkko 493 Foundation, AoF (grant numbers 336490 to O.V) and Helsinki University Hospital Funds 494 (TYH2018322).

\section{Author contributions}

496 P.J., L.K., J.L., A.K. and I.J. designed the experiments; P.J., P.K., M.H., S.M., R.L. and L.K. did 497 microneutralization tests and analyzed the data; P.J., A.R. and S.T. did EIA tests and analyzed the data; 498 H.K.H., S.H.P., P.T., I.L., A.N., T.M., H.V., L.I., J.L. and A.K. recruited vaccinees and patients and 499 collected their sera and data; A.P., R.N., P.J. and O.R. produced antigens for EIA; P.Ö., S.K., J.H. and 500 O.V. isolated and characterized virus strains; J.H. produced VeroE6-TMPRSS2-H10 cell line; T.S. did 501 sequencing and T.S., J.H. and P.K. analyzed sequences and structures; P.J. analyzed all data sets; P.J., 502 L.K. and I.J. wrote the manuscript and all co-authors contributed to the edition of the text.

\section{Competing Interests}

504 The authors declare no competing interests. 
506 Fig. 1 Antibody responses against SARS-CoV-2 S1 and N proteins in BNT162b2 vaccinated health care workers and non-hospitalized recovered COVID-19 patients.

508 A. Anti-S1 and B. anti-N IgG, $\operatorname{Ig}$ A, IgM, and total Ig antibody levels were measured with EIA. Serum 509 samples from BNT162b2 vaccinated participants $(\mathrm{n}=169)$ were collected before vaccination $(0 \mathrm{~d})$, and 510 three (3wk) and six (6wk) weeks after the first dose of the vaccine. All vaccinees received the second 511 dose of the vaccine three weeks after the first dose. Convalescent phase patient samples (Conv, $\mathrm{n}=50)$ 512 were collected 14 days to 6 weeks after the positive RT-qPCR test result. Data is represented as 513 geometric means and geometric standard deviations (SD). Cut-off values are indicated with dashed 514 lines.

\section{Fig. 2 Genetic analysis of virus variants and spike protein structure prediction.}

516 A. Schematic presentation of S gene and amino acid changes in FIN-25 (B.1 lineage), SR121

517 (B.1.463), 85HEL (B.1.1.7) and HEL12-102 (B.1.351) virus isolates used in the present study. B. 518 Trimeric SARS-CoV-2 S protein, referred to as the spike, structure in closed conformation (pdb: 519 6VXX). Amino acid substitutions (yellow) and deletions (green) as compared to the original spike 520 structure. C. Collective presentation of all amino acid changes found in virus isolates. Space filling 521 model indicating amino acids changes (yellow) and deletions (green) on the surface of a trimeric $\mathrm{S}$ 522 protein. Side and top views are shown.

523 Fig. 3 Neutralization of B.1.1.7 and B.1.351 variants by BNT162b2 vaccinees' sera and COVID52419 patient sera.

525 A. Neutralization titres for D614G variants FIN-25 and SR121, and 85HEL (B.1.1.7) and HEL12-102

526 (B.1.351) variants before (0d), three (3wk) and six weeks (6wk) after the first dose of BNT162b2 
527 vaccine and neutralization titres of convalescent sera of non-hospitalized patients. Values above the 528 groups indicate geometric mean titres (GMTs) and data is shown as geometric means and geometric 529 SDs. Neutralization titres $<20$ were plotted as 10 . B. Neutralization titres 3 weeks ( $3 w k)$ and six weeks

530 (6wk) after the first dose of the vaccine. Statistical differences between the virus isolates were analyzed 531 with Wilcoxon matched pairs signed-rank test. Two-tailed p-values $*<0.05, * *<0.01, * * * *<0.0001$ 532 were considered significant.

533 Fig. 4 Correlation of MNT titres against SARS-CoV-2 isolates.

534 MNT titres of BNT162b2 vaccinees $(\mathrm{n}=169)$ against FIN-25 were compared with MNT titres against 535 85HEL (B.1.1.7) and HEL12-102 (B.1.351) variants including 0d, 3wk and 6wk samples. Comparison 536 between two D614G virus isolates, FIN-25 and SR121, was done with sera from 86 BNT162b2 537 vaccinees. Correlation co-efficient ( $\mathrm{r}$ ) was calculated with Pearsons correlation test and p-values $<0.05$ 538 were considered significant. Each dot may represent multiple samples.

Fig. 5 Antibody responses against SARS-CoV-2 S1 protein and neutralization of B.1.1.7 and

\section{B.1.351 variants by age and gender.}

541 A. BNT162b2 vaccinated health care workers were divided into four age groups. Age specific 542 differences of anti-S1 IgG antibody levels and neutralization titres against FIN-25 virus isolate were 543 analyzed. Sera was collected three weeks (3w) and six weeks (6wk) after the first vaccine dose. B. 544 Gender-specific differences in antibody responses and neutralization titres. IgG antibody levels are 545 represented as EIA units. Differences between age and gender groups were tested with two-tailed 546 Mann-Whitney U test. Two-tailed p-values $*<0.05, * *<0.01, * * * * 0.0001$ were considered significant.

547 Fig. 6 Correlation of anti-S1 antibody levels with SARS-CoV-2 neutralization titres. 
548 Anti-S1 IgG and total Ig antibody levels were determined with EIA and neutralization titres of 549 BNT162b2 vaccinated health care workers $(n=169)$ against FIN-25 virus isolate were obtained with 550 microneutralization test (MNT). All sequential serum samples (0d, 3wk and 6wk) were included in the 551 analysis. Spearman's rank correlation coefficient (r) is indicated. 
552 Table 1 Antibody responses in BNT162b2 vaccinated health care workers (HCW) and non-

553 hospitalized convalescent phase COVID-19 patients. HCW samples were collected before

554 vaccination (0d), and three ( $3 \mathrm{wk})$ and six (6wk; three weeks after the second vaccine dose) weeks after 555 the first vaccine dose. Geometric mean (GM) and number of positive samples for anti-S1 IgG and total 556 Ig, and anti-N IgG antibodies and neutralizing antibodies is indicated. In microneutralization test

557 (MNT) neutralization titre $>20$ was considered positive and for calculation of geometric means a value 558 of 10 was given for values of $<20$.

\begin{tabular}{|c|c|c|c|c|c|c|c|c|c|}
\hline & & \multicolumn{2}{|r|}{ Od } & \multicolumn{2}{|r|}{$3 w k$} & \multicolumn{2}{|r|}{ 6wk } & \multicolumn{2}{|c|}{ Convalescent } \\
\hline & & GM & Positive (n/n) & GM & Positive (n/n) & GM & Positive (n/n) & GM & Positive (n/n) \\
\hline \multirow[t]{3}{*}{ EIA } & anti-S1 IgG & 1 & $0 \%(0 / 169)$ & 47 & $96 \%(160 / 167)$ & 107 & $100 \%(169 / 169)$ & 20 & $62 \%(31 / 50)$ \\
\hline & anti-S1 tot Ig & 2 & $4 \%(6 / 169)$ & 37 & $96 \%(160 / 167)$ & 86 & $100 \%(169 / 169)$ & 23 & $82 \%(41 / 50)$ \\
\hline & anti-N IgG & 2 & $4 \%(6 / 169)$ & 2 & $7 \%(11 / 167)$ & 2 & $5 \%(9 / 169)$ & 17 & $66 \%(33 / 50)$ \\
\hline \multirow[t]{4}{*}{ MNT } & FIN-25 & 10 & $0 \%(0 / 169)$ & 24 & $63 \%(106 / 167)$ & 234 & $100 \%(169 / 169)$ & 55 & $86 \%(43 / 50)$ \\
\hline & SR121 & 10 & $0 \%(0 / 84)$ & 32 & $83 \%(70 / 84)$ & 275 & $100 \%(86 / 86)$ & 86 & $96 \%(48 / 50)$ \\
\hline & 85HEL & 10 & $0 \%(1 / 169)$ & 24 & $63 \%(106 / 167)$ & 240 & $100 \%(169 / 169)$ & 74 & $96 \%(48 / 50)$ \\
\hline & HEL12-102 & 10 & $0 \%(0 / 169)$ & 12 & $15 \%(25 / 167)$ & 48 & $92 \%(156 / 169)$ & 16 & $56 \%(28 / 50)$ \\
\hline
\end{tabular}



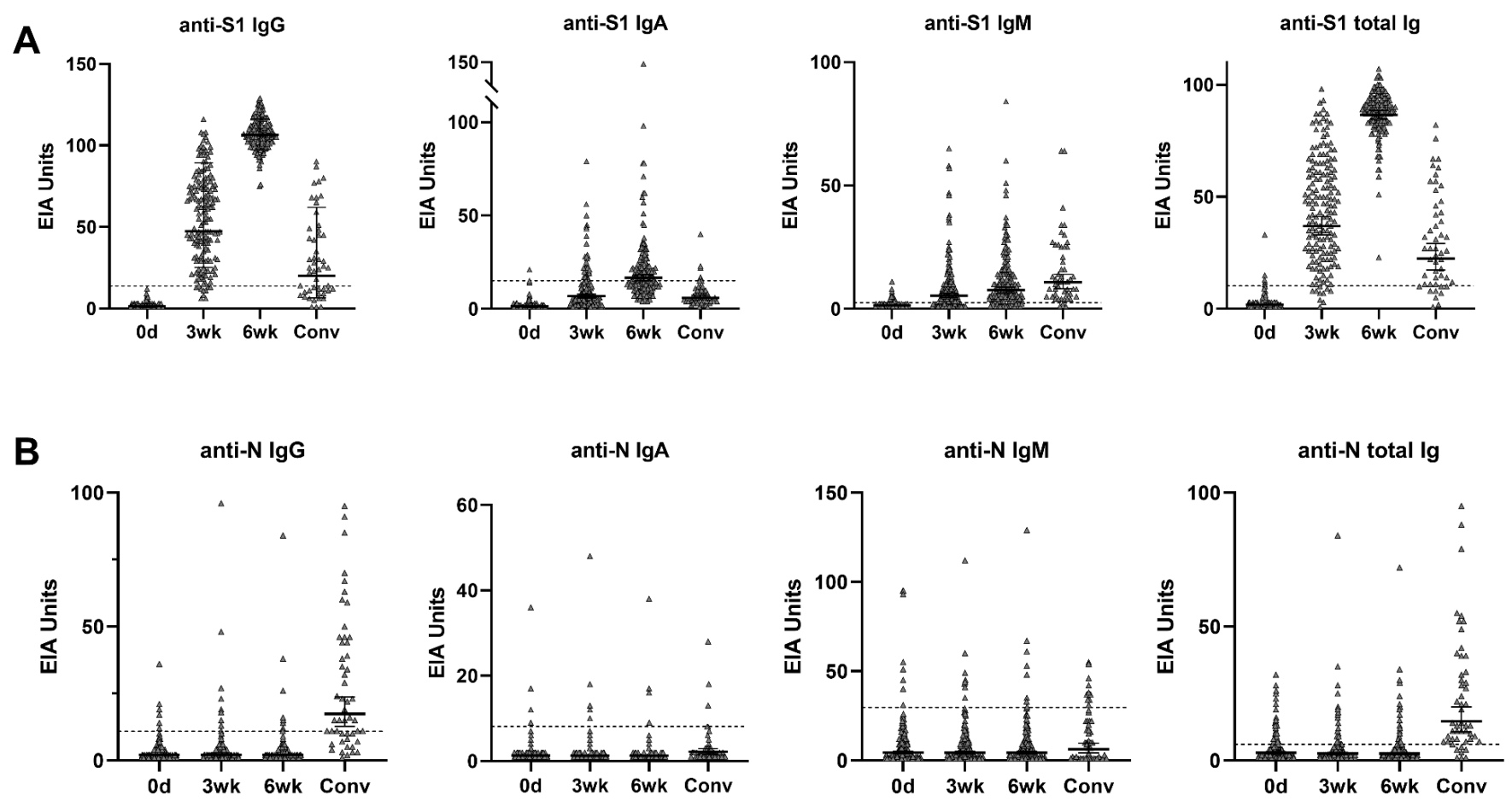

561

562 


\section{$563 \quad$ Figure 2}
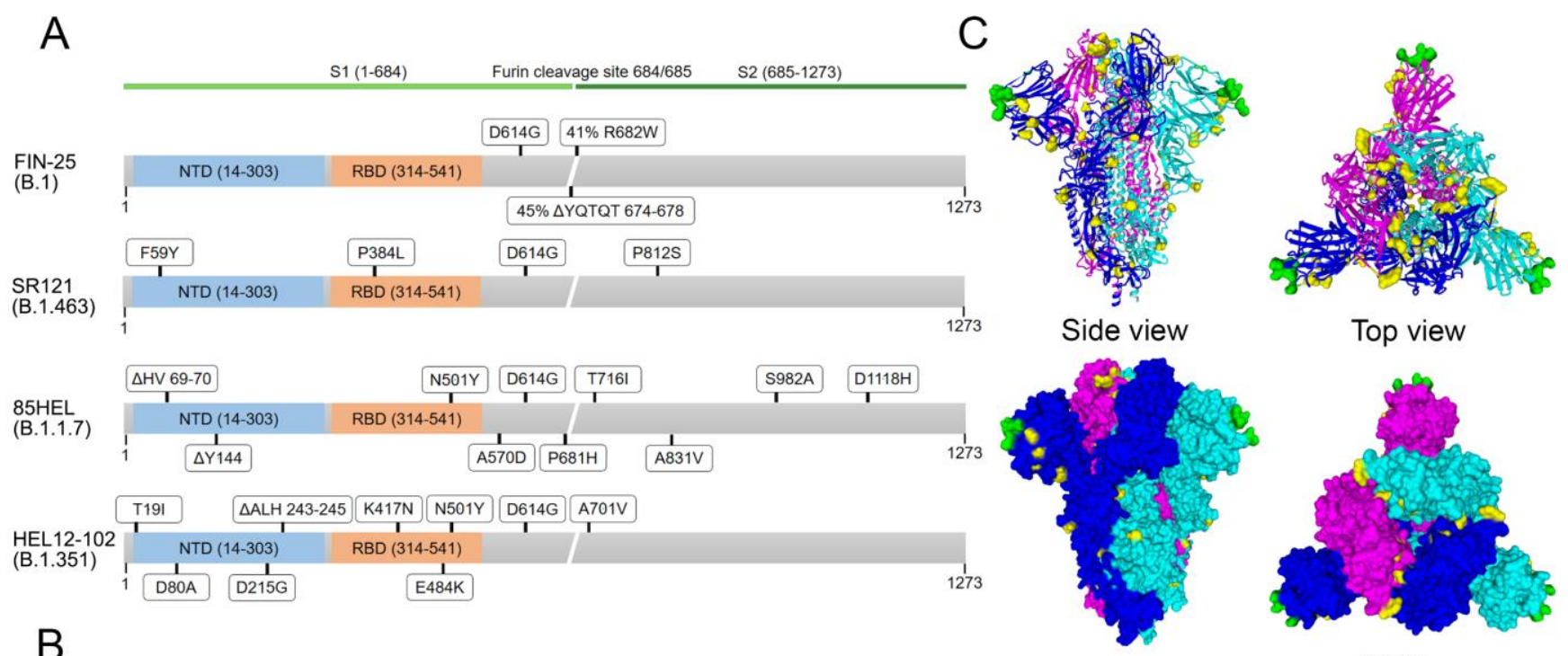

B

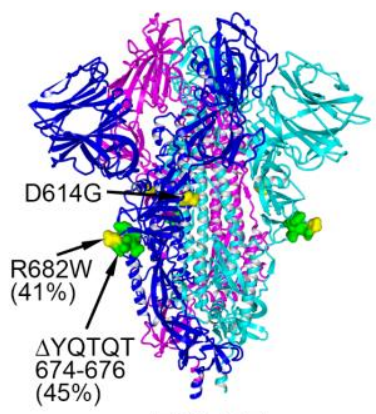

FIN-25

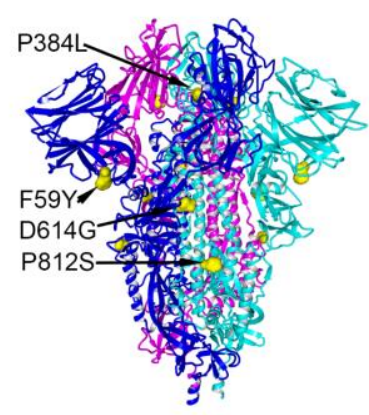

SR121
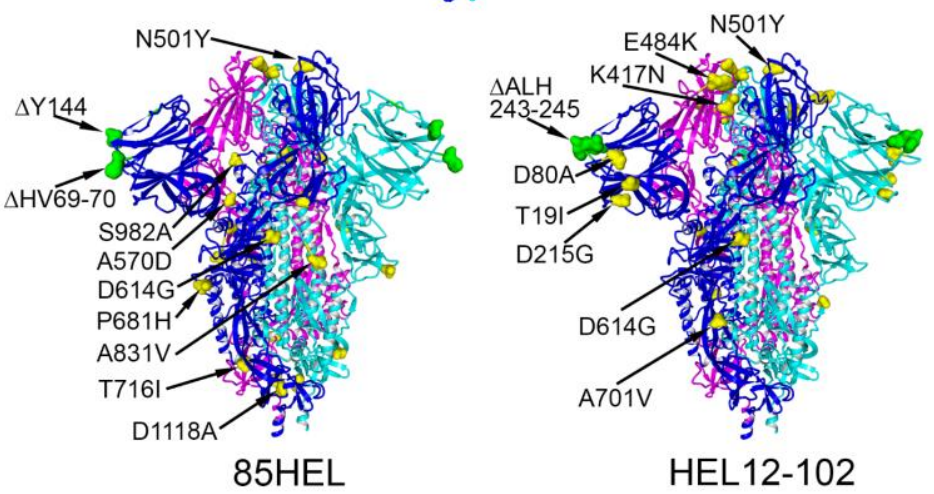

HEL12-102 
A

FIN-25

(B.1)

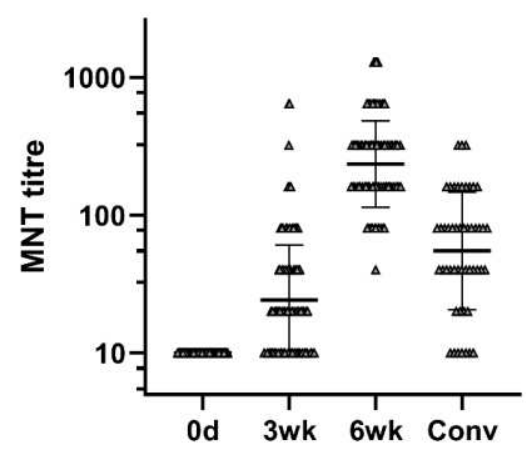

85HEL

(B.1.1.7)

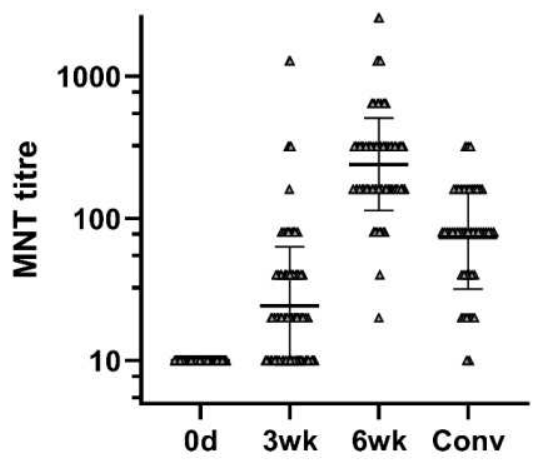

B

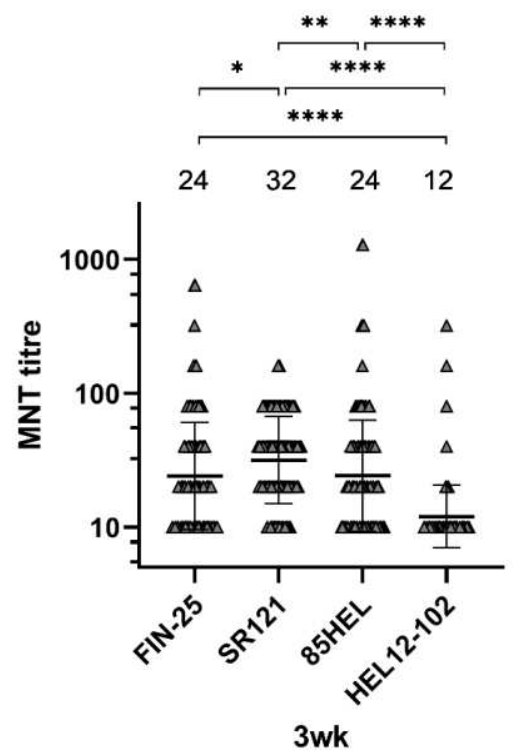

SR121

(B.1.463)

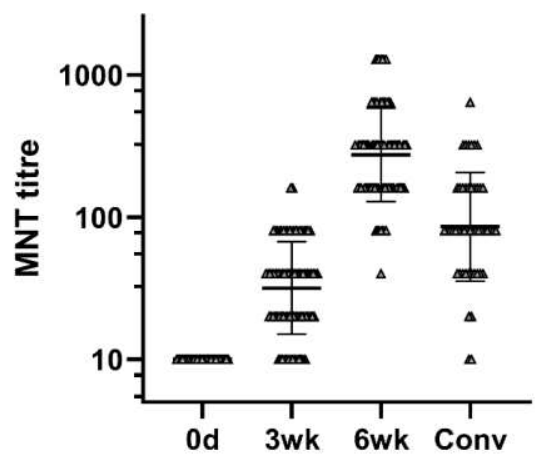

HEL12-102

(B.1.351)
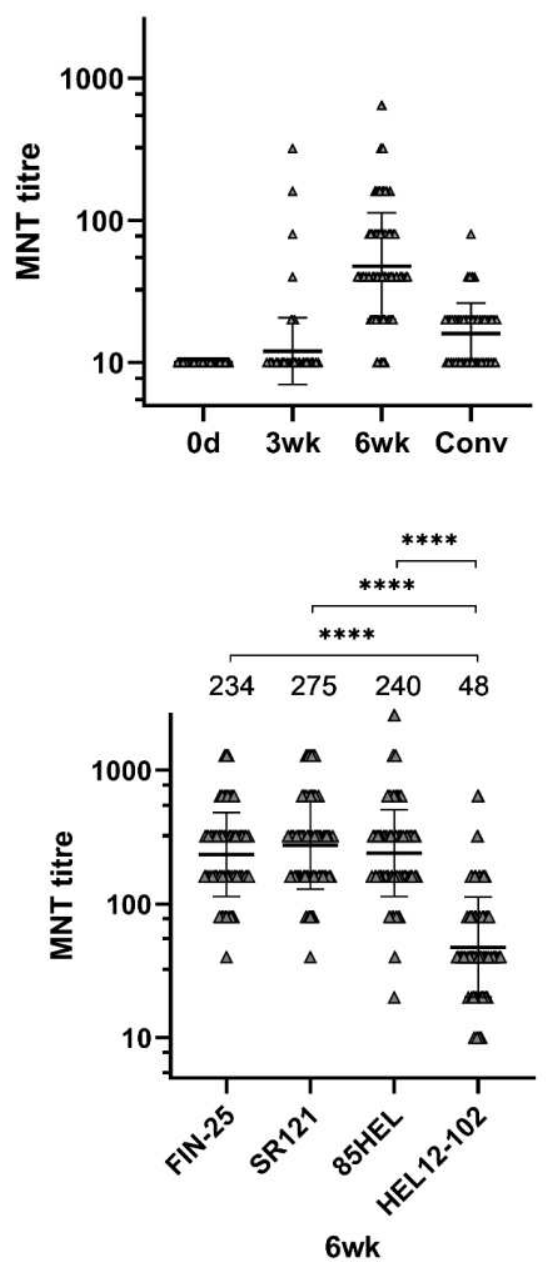

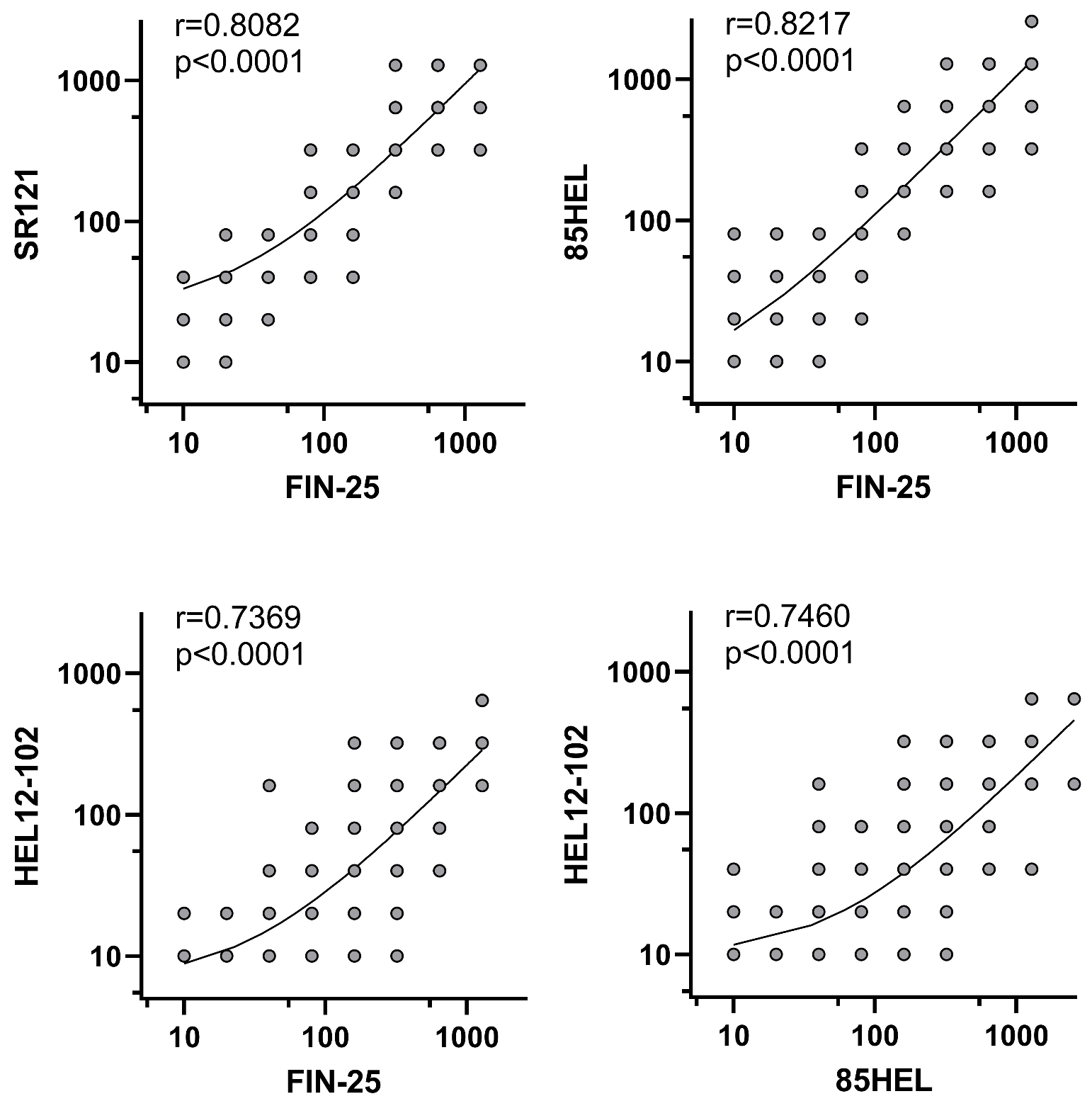

569

570 
A
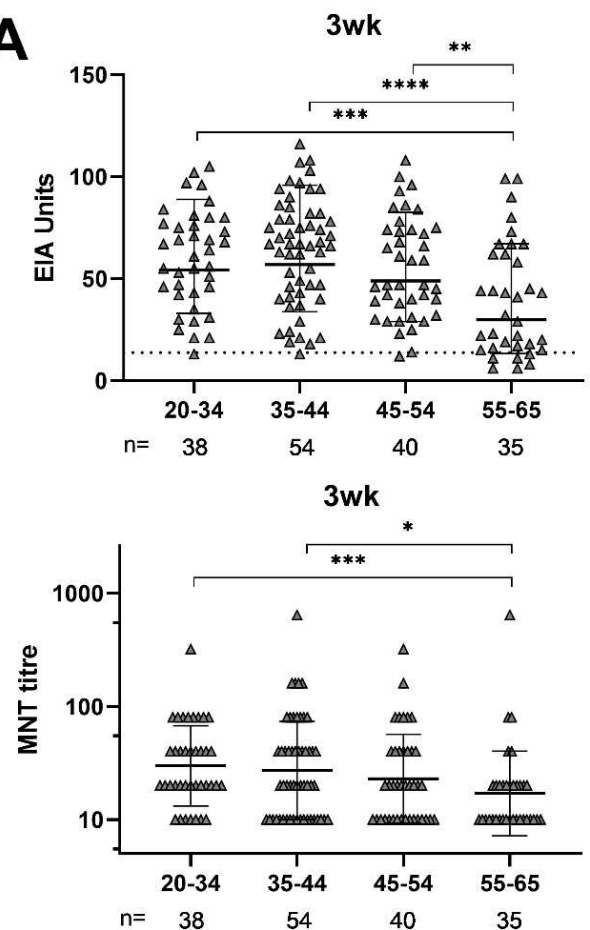
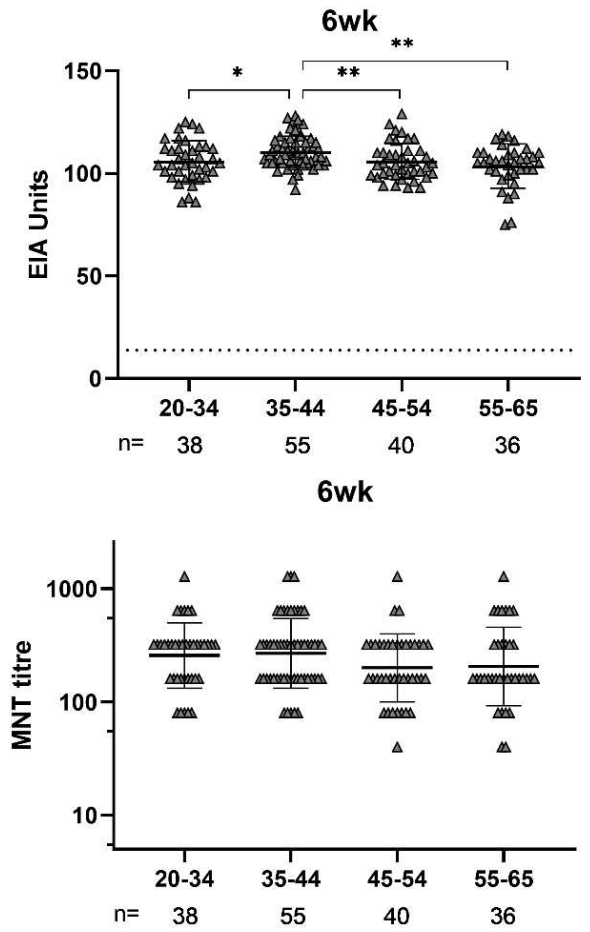

B
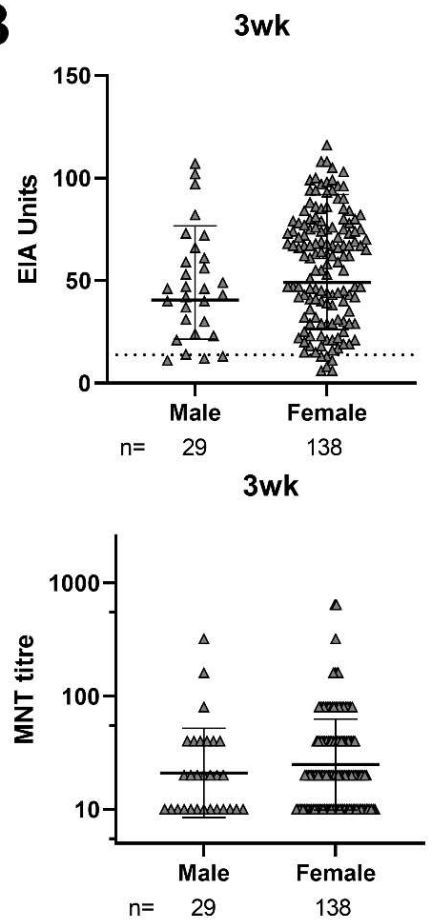

6wk
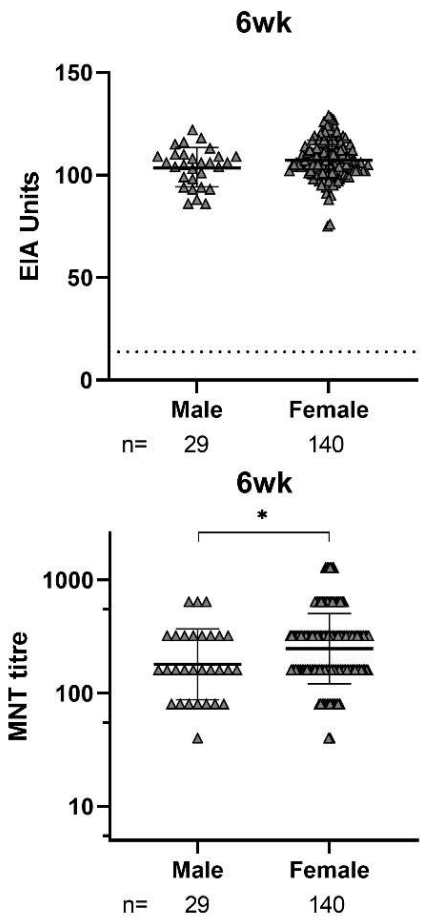

572 
$573 \quad$ Figure 6
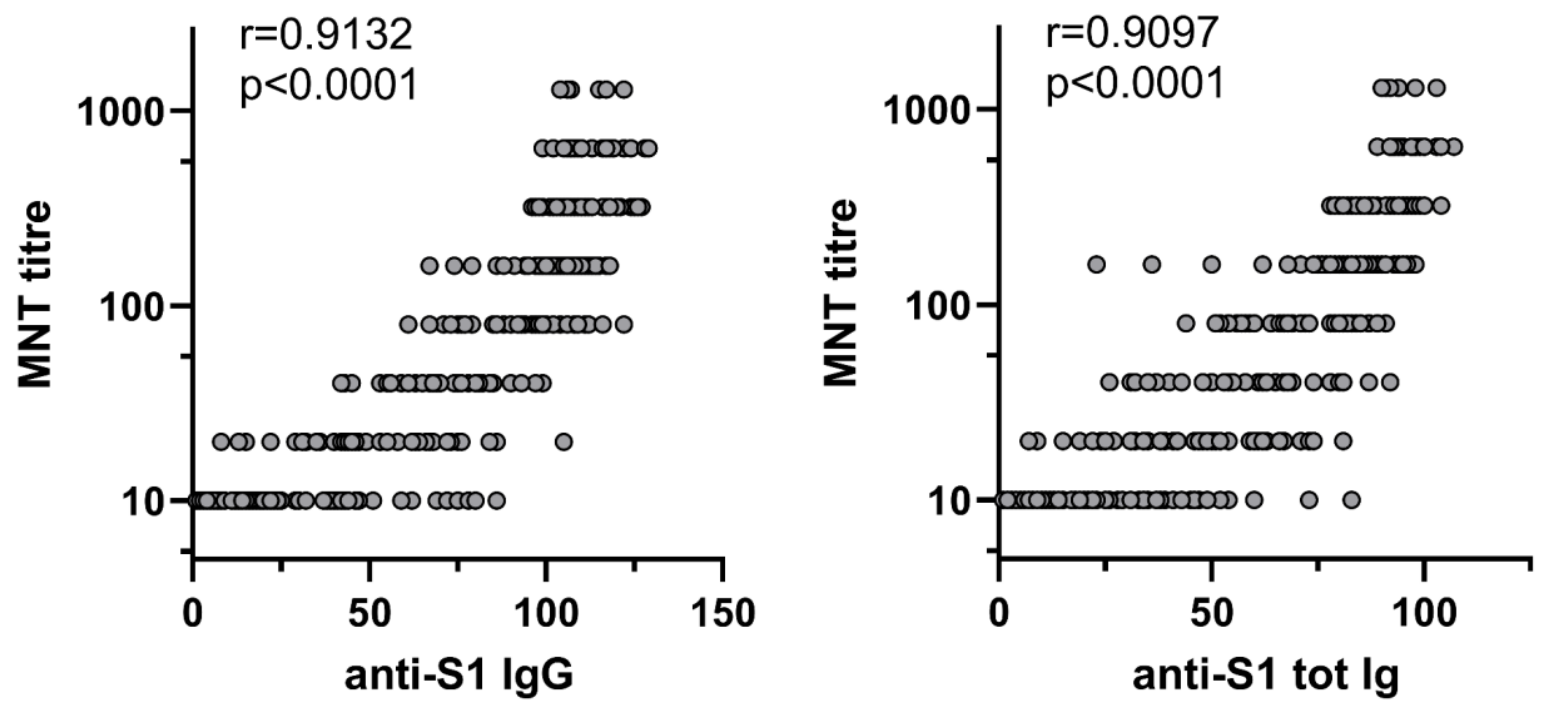

574 
Figures

\section{Figure 1}
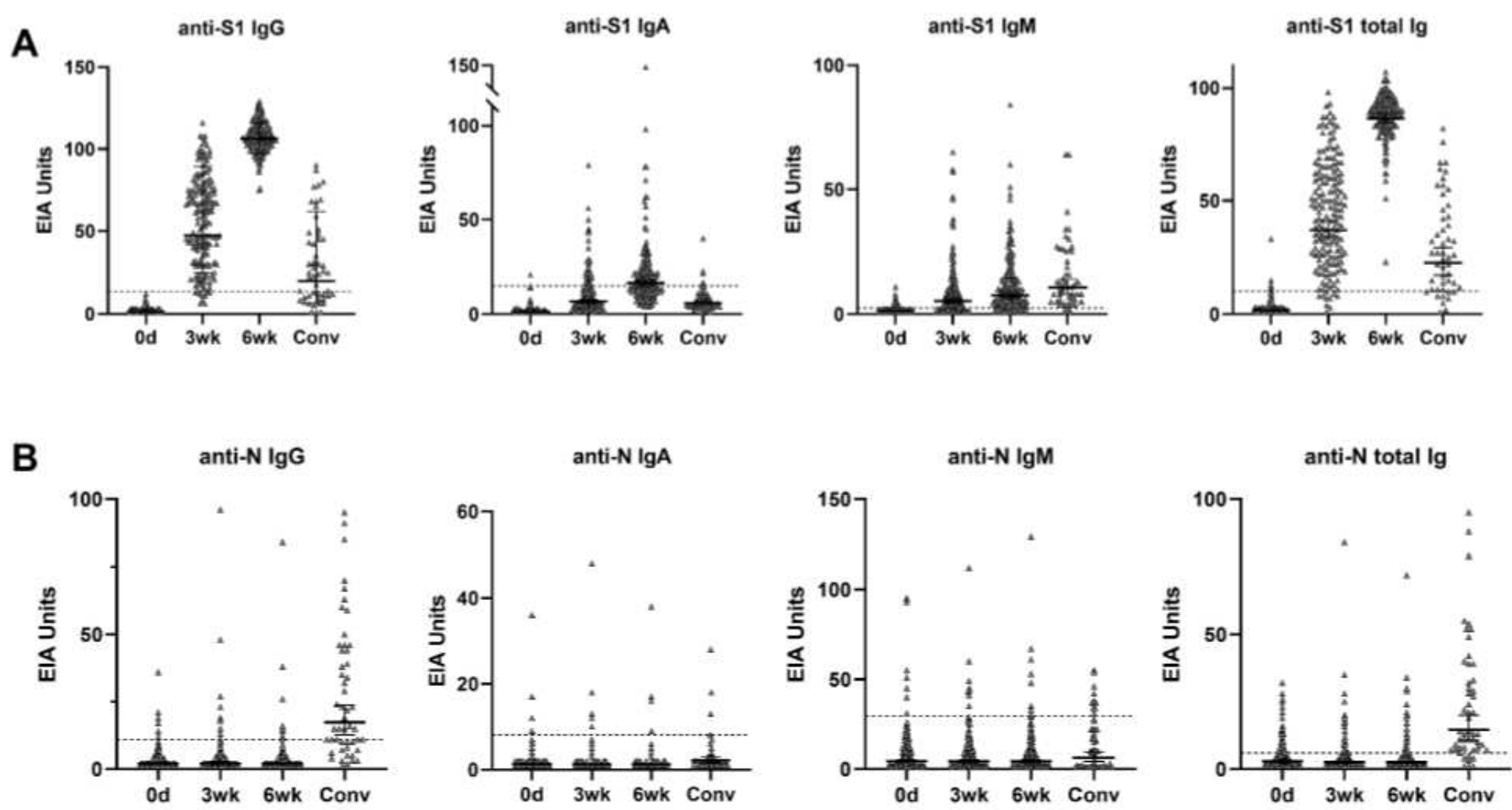

\section{Figure 1}

Antibody responses against SARS-CoV-2 S1 and N proteins in BNT162b2 vaccinated health care workers and non-hospitalized recovered COVID-19 patients. A. Anti-S1 and B. anti-N IgG, IgA, IgM, and total Ig antibody levels were measured with EIA. Serum samples from BNT162b2 vaccinated participants $(n=169)$ were collected before vaccination $(0 d)$, and three (3wk) and six (6wk) weeks after the first dose of the vaccine. All vaccinees received the second dose of the vaccine three weeks after the first dose. Convalescent phase patient samples (Conv, $n=50$ ) were collected 14 days to 6 weeks after the positive RTqPCR test result. Data is represented as geometric means and geometric standard deviations (SD). Cutoff values are indicated with dashed lines. 


\section{Figure 2}

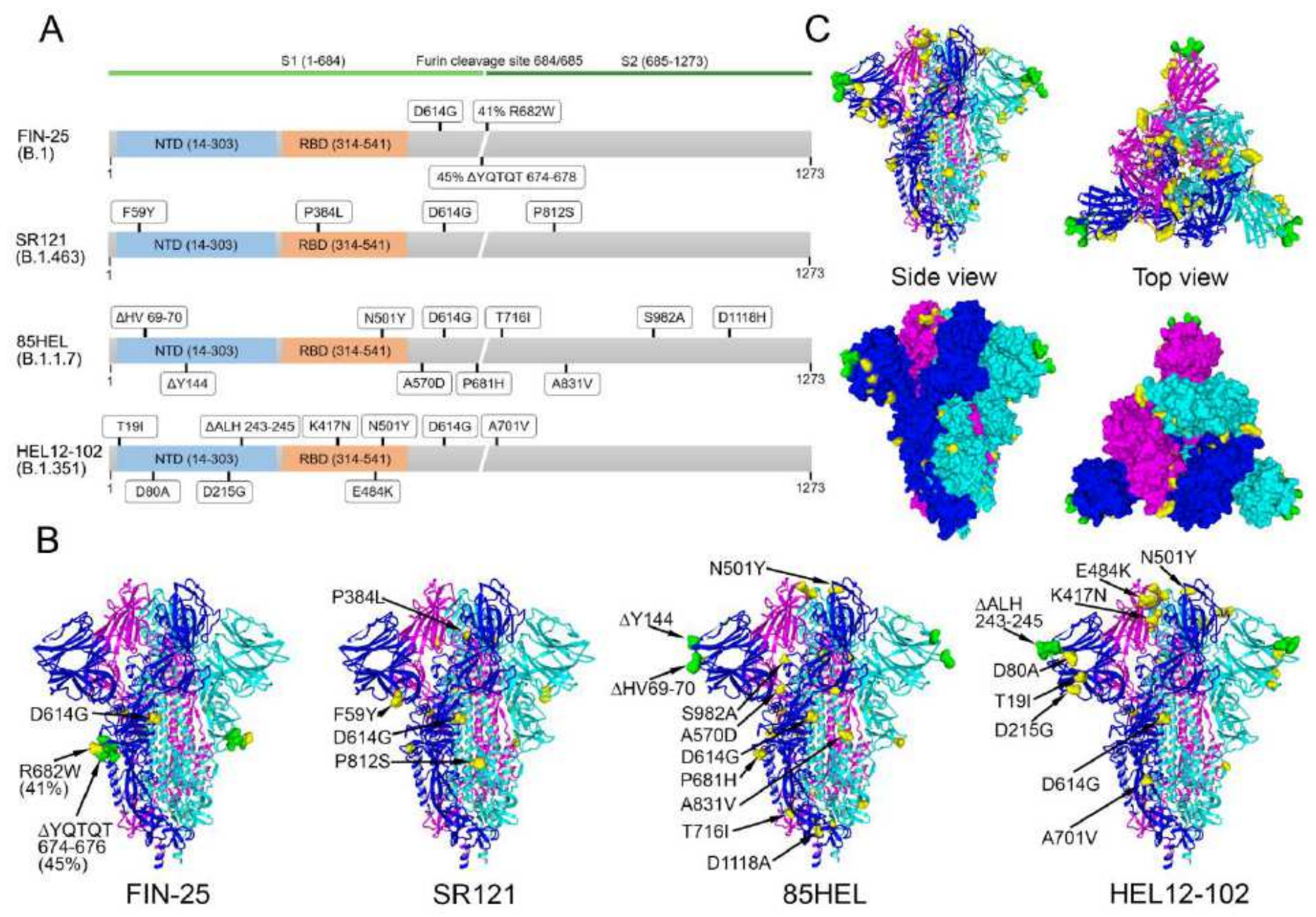

Figure 2

Genetic analysis of virus variants and spike protein structure prediction. A. Schematic presentation of $S$ gene and amino acid changes in FIN-25 (B.1 lineage), SR121 (B.1.463), 85HEL (B.1.1.7) and HEL12-102 (B.1.351) virus isolates used in the present study. B. Trimeric SARS-CoV-2 S protein, referred to as the spike, structure in closed conformation (pdb: 6VXX). Amino acid substitutions (yellow) and deletions (green) as compared to the original spike structure. C. Collective presentation of all amino acid changes found in virus isolates. Space filling model indicating amino acids changes (yellow) and deletions (green) on the surface of a trimeric S protein. Side and top views are shown. 
Figure 3

A

FIN-25

(B.1)

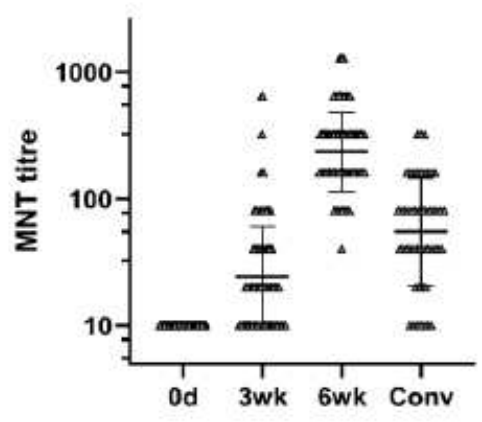

85HEL

(B.1.1.7)

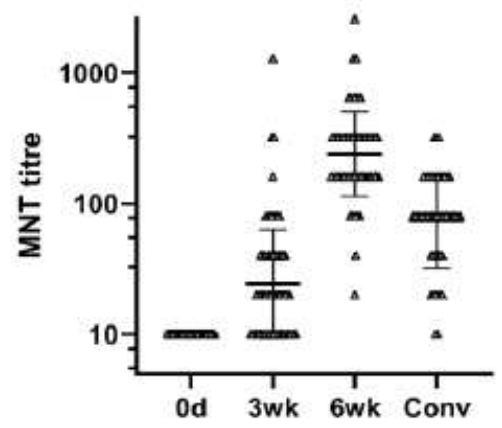

B

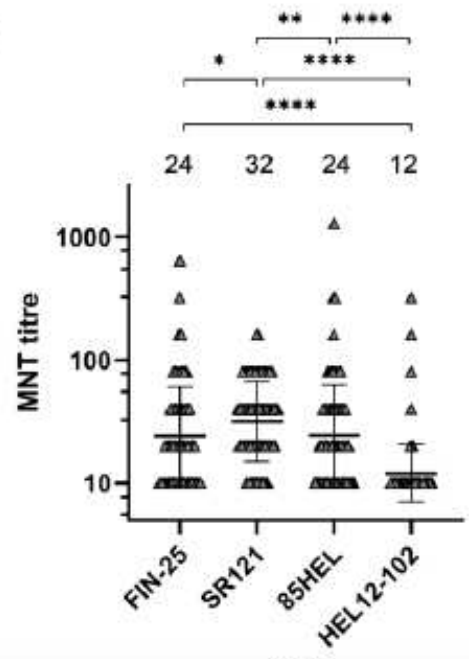

3wk
SR121

(B.1.463)

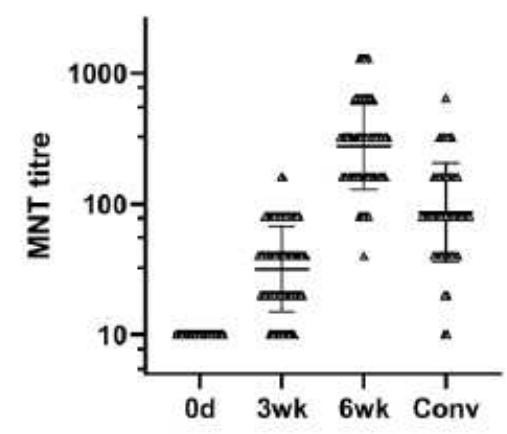

HEL12-102

(B.1.351)
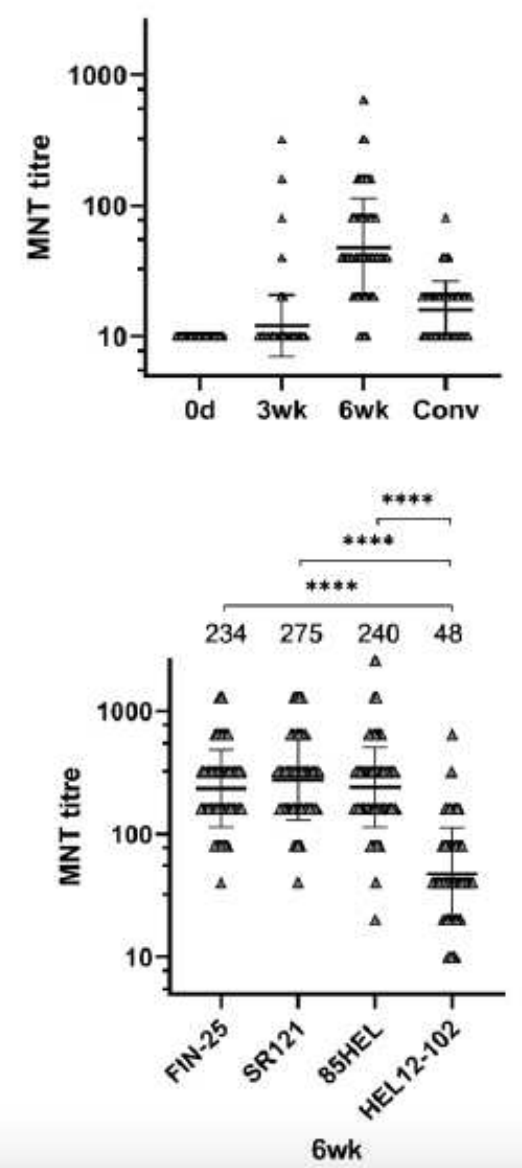

\section{Figure 3}

Neutralization of B.1.1.7 and B.1.351 variants by BNT162b2 vaccinees' sera and COVID 19 patient sera. A. Neutralization titres for D614G variants FIN-25 and SR121, and 85HEL (B.1.1.7) and HEL12-102 (B.1.351) variants before (0d), three (3wk) and six weeks (6wk) after the first dose of BNT162b2 vaccine and neutralization titres of convalescent sera of non-hospitalized patients. Values above the groups indicate geometric mean titres (GMTs) and data is shown as geometric means and geometric SDs. 
Neutralization titres $<20$ were plotted as 10 . B. Neutralization titres 3 weeks ( $3 w k)$ and six weeks $(6 w k)$ after the first dose of the vaccine. Statistical differences between the virus isolates were analyzed with Wilcoxon matched pairs signed-rank test. Two-tailed p-values $*<0.05, * *<0.01, * \star * *<0.0001$ were considered significant

\section{Figure 4}
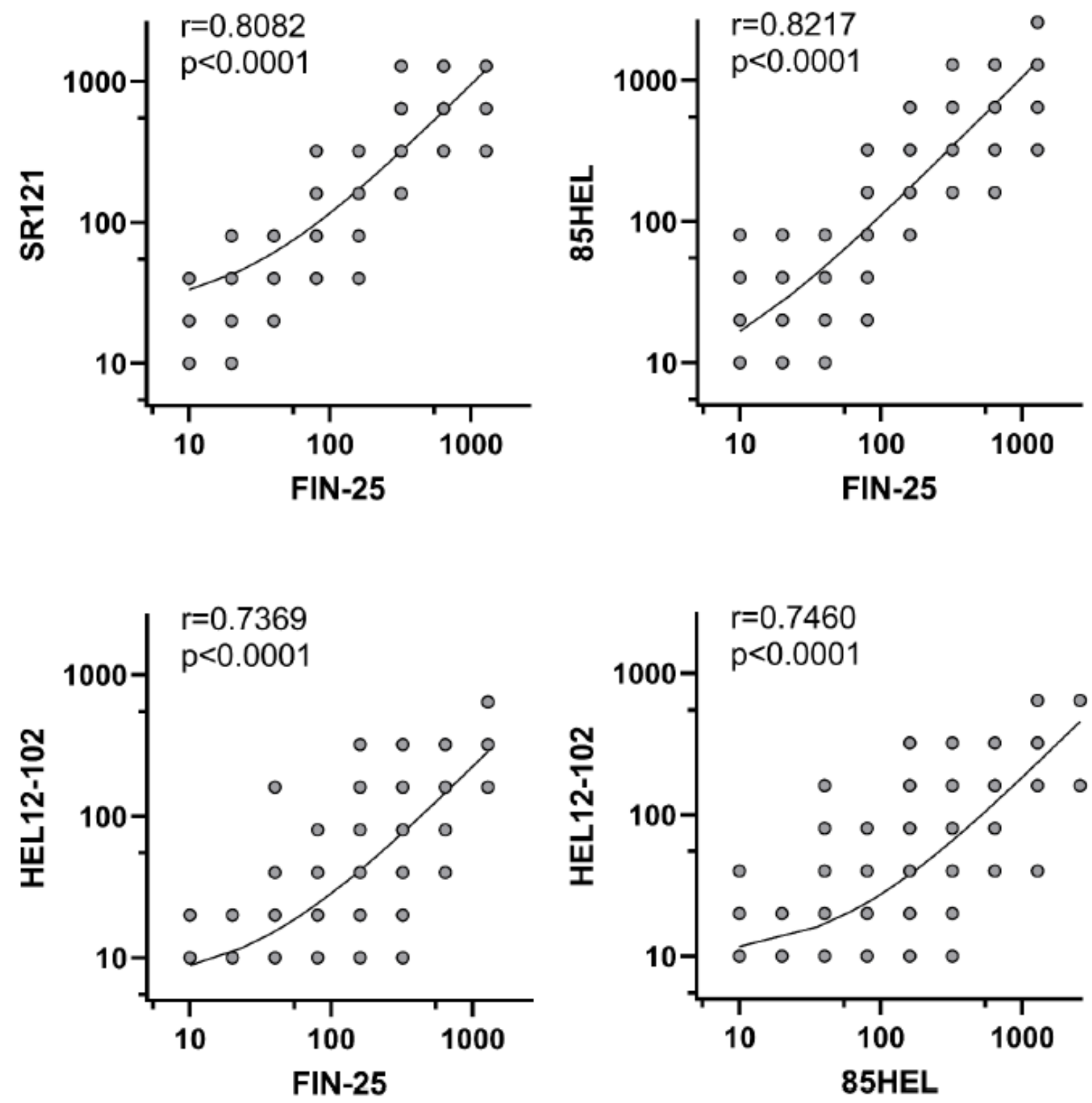

Figure 4 
including 0d, 3wk and 6wk samples. Comparison between two D614G virus isolates, FIN-25 and SR121, was done with sera from 86 BNT162b2 vaccinees. Correlation co-efficient ( $r$ ) was calculated with Pearsons correlation test and $p$-values $<0.05$ were considered significant. Each dot may represent multiple samples.

\section{Figure 5}

A
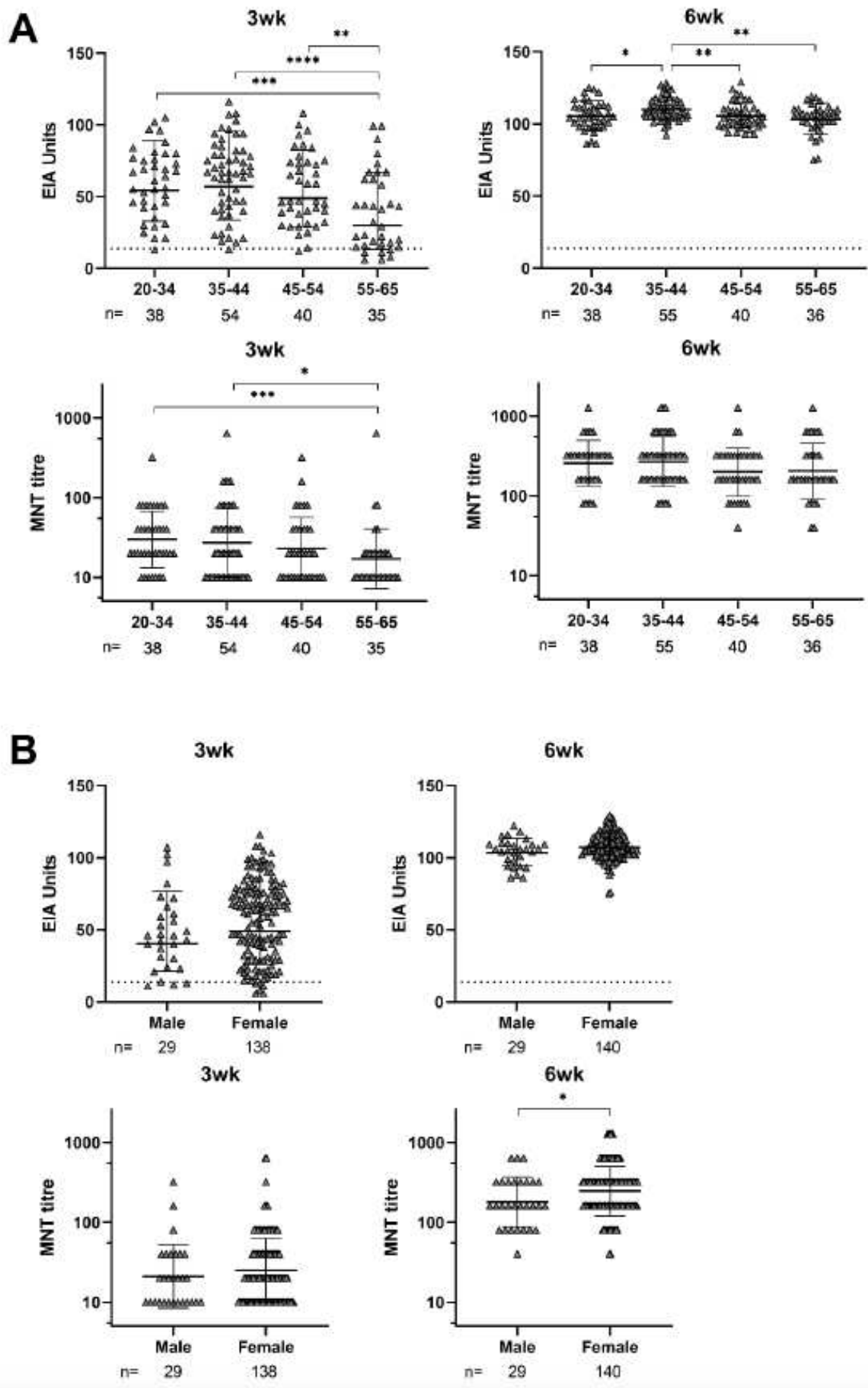

Figure 5 
Antibody responses against SARS-CoV-2 S1 protein and neutralization of B.1.1.7 and B.1.351 variants by age and gender. A. BNT162b2 vaccinated health care workers were divided into four age groups. Age specific differences of anti-S1 IgG antibody levels and neutralization titres against FIN-25 virus isolate were analyzed. Sera was collected three weeks $(3 w)$ and six weeks $(6 w k)$ after the first vaccine dose. B. Gender-specific differences in antibody responses and neutralization titres. IgG antibody levels are represented as EIA units. Differences between age and gender groups were tested with two-tailed MannWhitney U test. Two-tailed p-values $*<0.05, * *<0.01, * * * *<0.0001$ were considered significant.

\section{Figure 6}
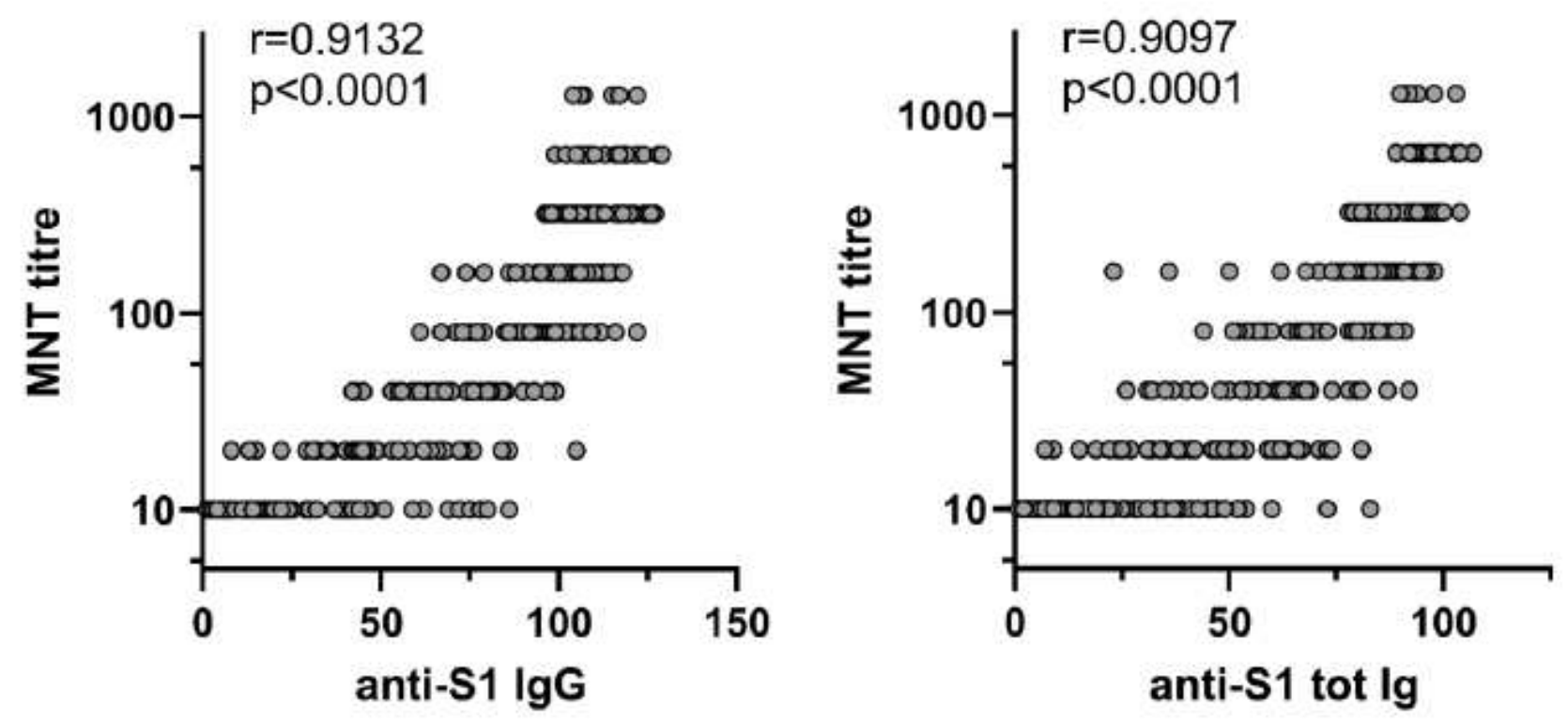

Figure 6

Correlation of anti-S1 antibody levels with SARS-CoV-2 neutralization titres. Anti-S1 IgG and total Ig antibody levels were determined with EIA and neutralization titres of BNT162b2 vaccinated health care workers ( $n=169)$ against FIN-25 virus isolate were obtained with microneutralization test (MNT). All sequential serum samples (0d, 3wk and $6 \mathrm{wk}$ ) were included in the analysis. Spearman's rank correlation coefficient $(r)$ is indicated.

\section{Supplementary Files}

This is a list of supplementary files associated with this preprint. Click to download.

- Supplementarylnformation.pdf 